\title{
A FICTITIOUS DOMAIN METHOD FOR THE NUMERICAL TWO-DIMENSIONAL SIMULATION OF POTENTIAL FLOWS PAST SAILS*
}

\author{
Alfredo Bermúdez ${ }^{1}$, Rodolfo Rodríguez ${ }^{2}$ And María Luisa Seohne ${ }^{1}$
}

\begin{abstract}
This paper deals with the mathematical and numerical analysis of a simplified twodimensional model for the interaction between the wind and a sail. The wind is modeled as a steady irrotational plane flow past the sail, satisfying the Kutta-Joukowski condition. This condition guarantees that the flow is not singular at the trailing edge of the sail. Although for the present analysis the position of the sail is taken as data, the final aim of this research is to develop tools to compute the sail shape under the aerodynamic pressure exerted by the wind. This is the reason why we propose a fictitious domain formulation of the problem, involving the wind velocity stream function and a Lagrange multiplier; the latter allows computing the force density exerted by the wind on the sail. The Kutta-Joukowski condition is imposed in integral form as an additional constraint. The resulting problem is proved to be well posed under mild assumptions. For the numerical solution, we propose a finite element method based on piecewise linear continuous elements to approximate the stream function and piecewise constant ones for the Lagrange multiplier. Error estimates are proved for both quantities and a couple of numerical tests confirming the theoretical results are reported. Finally the method is used to determine the sail shape under the action of the wind.
\end{abstract}

Mathematics Subject Classification. 65N30, 76M10.

Received March 19, 2010. Revised January 19, 2011.

Published online June 13, 2011

\section{INTRODUCTION}

In the last years the competition in nautical sports, as the America's Cup, has been the source of many important developments in mechanical engineering. The computer aided design is the key to the most efficient use of the wind force and steers the configuration of the hull and the sails in order to reach a greater speed.

The aim of this paper is the mathematical analysis of the interaction between the wind and a sail and its numerical solution. A large amount of research has been devoted to model and solve the flow past a sharp obstacle with changeable outline. This problem deals with numerous application domains, yacht and aircraft

\footnotetext{
Keywords and phrases. Finite element approximation, fluid-structure interaction, fictitious domain, flow past sails.

* Research partially supported by Ministerio de Ciencia e Innovación (Spain) under research projects Consolider MATHEMATICA CSD2006-00032 and MTM2008-02483, Xunta de Galicia-FEDER research project CEUIC 2010-22 and FONDAP and BASAL projects CMM, Universidad de Chile (Chile).

1 Departamento de Matemática Aplicada, Universidad de Santiago de Compostela, 15706, Santiago de Compostela, Spain. alfredo.bermudez@usc.es; marialuisa.seoane@usc.es

${ }^{2} \mathrm{CI}^{2} \mathrm{MA}$, Departamento de Ingeniería Matemática, Universidad de Concepción, Casilla 160-C, Concepción, Chile.

rodolfo@ing-mat.udec.cl
} 
design among others. But, even if the aerodynamics are very close, there is a notorious difference: the sails, contrary to airfoils, are flexible structures. Therefore, the flow around the sail depends upon its shape, whereas the shape results from the aerodynamic pressure exerted by the wind. The whole problem is very complex, both from the physical and the mathematical points of view. On the one hand, the more sophisticated fluid models include viscous effects and the laminar-turbulent transition near the sail, which is considered a curved elastic membrane subjected to large displacements; on the other hand, these two nonlinear models are coupled in a fluid-structure problem. Moreover, apart from the theoretical complexity, their computational solution becomes highly expensive, as is pointed out by Parolini and Quarteroni [11].

The first mathematical model where the sail and the pressure are considered as unknowns was developed by Thwaites [14] in the framework of the two-dimensional potential flow theory. It involves a linear model of an elastic flexible sail and an integral equation which expresses that the tension due to the sail curvature is exactly counterbalanced by the aerodynamic pressure. More recently, an extension to the three dimensional case was made by Schoop [12] involving the Navier-Stokes equations for flow simulation. Related to the structural sail problem, Muttin [10] proposed a nonlinear membrane model in large displacements and small strains, which he solved by a finite element method. However, the approach in all these papers is merely computational and no rigorous mathematical or numerical analysis of the models is intended. On the other hand, a mathematical study of steady irrotational plane flows past profiles with a sharp trailing edge was done by Ciavaldini et al. [3]. In particular, they proposed a rigorous mathematical formulation of the classical Kutta-Joukowski condition.

The present paper is somehow inspired in this article. As in this reference the analysis is restricted to a two-dimensional potential flow, but we consider the case of a sail section instead of a wing profile. Thus, the corresponding plane flow is singular at the point corresponding to the leading edge, which makes the mathematical analysis more involved. Moreover, we envisage to consider the full fluid-structure problem, i.e., computing the sail shape rather than giving it as a data of the problem. This is why we propose a fictitious domain method for the numerical solution. Thus, we will be able to avoid remeshing the fluid domain when the sail shape changes along the iterations. The analysis of this method follows essentially the papers by Glowinski et al. [7] and Girault and Glowinski [5]. However, as explained above, we have to adapt these techniques because of the lack of regularity of the solution, due to the singularities on the boundary of the flow domain.

The outline of the paper is as follows. After this introduction we state the flow model to be considered along this paper in Section 1. In particular, we formulate the Kutta-Joukowski condition as an integral constraint guaranteeing that the flow is not singular at the trailing edge of the sail. The mathematical analysis of the whole model is performed in Section 2 for a fictitious domain formulation involving the stream function and a Lagrange multiplier defined on the sail. Although the full problem does not fall into the Babuška-Brezzi theory of mixed formulations, the latter is the main functional tool to prove the existence and uniqueness of a solution under mild assumptions. Section 3 is devoted to the numerical solution. A finite element discretization involving continuous piecewise linear functions to approximate the stream function and piecewise constant ones for the Lagrange multiplier on the sail is proposed. Appropriate error estimates are obtained for the approximations of both functions. In Section 4 we report numerical results for a couple of test problems which confirm the theoretical results. Finally we apply the numerical method to solve the fluid-structure interaction problem of determining the shape of a sail under the action of the wind.

\section{The mathematical Model}

In this paper we study a simplified flow model similar to the one considered in [14] (see also [3]): a twodimensional flow past a sail modeled as a plane curve $\mathcal{S}$.

Let us denote by $\boldsymbol{x}_{\mathrm{L}}$ and $\boldsymbol{x}_{\mathrm{T}}$ the two end points of $\mathcal{S}$. More specifically, let $\boldsymbol{x}_{\mathrm{L}}$ be the leading point and $\boldsymbol{x}_{\mathrm{T}}$ be the trailing point of the sail. Let us denote the uniform flow velocity at infinity by $\boldsymbol{v}_{\infty}$. For the sake of simplicity and having in view the numerical solution of the problem by finite element methods, we suppose the flow takes place in a large enough bounded domain $\Omega$, with a Lipschitz-continuous polygonal exterior boundary $\Gamma$ and such that $\mathcal{R}:=\Omega \cup \mathcal{S}$ is convex (see Fig. 1). 


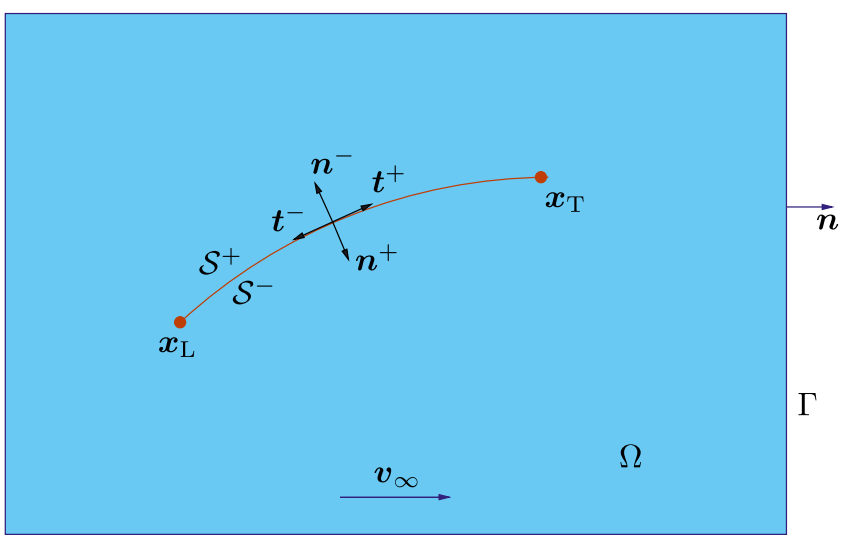

Figure 1. Flow domain.

We assume the fluid is inviscid and incompressible, and the flow is steady, so that it is governed by the steady incompressible Euler equations, namely,

$$
\begin{aligned}
\rho(\operatorname{grad} \boldsymbol{v}) \boldsymbol{v}+\operatorname{grad} \pi & =\boldsymbol{b} & & \text { (motion equation }), \\
\operatorname{div} \boldsymbol{v} & =0 & & \text { (incompressibility equation }),
\end{aligned}
$$

where $\rho$ is the (constant) density, $\boldsymbol{v}$ the velocity, $\pi$ the pressure and $\boldsymbol{b}$ a body force. This is a first order nonlinear hyperbolic system of partial differential equations. However, assuming that the body force is conservative (i.e., $\boldsymbol{b}=-\rho \operatorname{grad} \phi$ for some scalar potential $\phi$ ) and the flow is irrotational (i.e., curl $\boldsymbol{v}=0$ ), it is well-known that these equations are fully equivalent to the following simpler ones (see for instance [9]):

$$
\begin{aligned}
\operatorname{curl} \boldsymbol{v} & =0, \\
\operatorname{div} \boldsymbol{v} & =0, \\
\frac{|\boldsymbol{v}|^{2}}{2}+\frac{\pi}{\rho}+\phi & =\text { const. }
\end{aligned}
$$

The latter is the Bernoulli's equation.

To either of the above systems, the following boundary conditions will be added:

$$
\begin{aligned}
\boldsymbol{v} \cdot \boldsymbol{n}=0 & \text { on } \mathcal{S}, \\
\boldsymbol{v} \cdot \boldsymbol{n}=\boldsymbol{v}_{\infty} \cdot \boldsymbol{n} & \text { on } \Gamma .
\end{aligned}
$$

Here and thereafter $\boldsymbol{n}$ denotes a unit vector normal to a particular curve, which will be clear from the context. When the curve is the boundary of a bounded domain, $\boldsymbol{n}$ is always taken as the outward normal.

Let $\left\{\boldsymbol{e}_{1}, \boldsymbol{e}_{2}\right\}$ be an orthonormal basis of $\mathbb{R}^{2}$. We denote by $\mathcal{S}^{+}$and $\mathcal{S}^{-}$the two sides of the sail and by $\boldsymbol{t}^{ \pm}=t_{1}^{ \pm} \boldsymbol{e}_{1}+t_{2}^{ \pm} \boldsymbol{e}_{2}$ (respectively, $\boldsymbol{n}^{ \pm}=t_{2}^{ \pm} \boldsymbol{e}_{1}-t_{1}^{ \pm} \boldsymbol{e}_{2}$ ) the unit tangent (respectively, unit normal) vector to $\mathcal{S}^{ \pm}$, chosen as shown in Figure 1.

It is possible to obtain an approximation of the force acting at each point of the sail $\mathcal{S}$, which can be used to determine the sail shape, as follows (see [14]):

$$
\begin{aligned}
\boldsymbol{f} & :=\pi^{+} \boldsymbol{n}^{+}+\pi^{-} \boldsymbol{n}^{-}=-\rho \frac{\left|\boldsymbol{v}^{+}\right|^{2}}{2} \boldsymbol{n}^{+}-\rho \frac{\left|\boldsymbol{v}^{-}\right|^{2}}{2} \boldsymbol{n}^{-}=\rho\left(\boldsymbol{v}^{+} \cdot \boldsymbol{t}^{+}+\boldsymbol{v}^{-} \cdot \boldsymbol{t}^{-}\right) \frac{\boldsymbol{v}^{+} \cdot \boldsymbol{t}^{+}-\boldsymbol{v}^{-} \cdot \boldsymbol{t}^{-}}{2} \boldsymbol{n}^{-} \\
& \approx \rho v_{\infty}\left(\boldsymbol{v}^{+} \cdot \boldsymbol{t}^{+}+\boldsymbol{v}^{-} \cdot \boldsymbol{t}^{-}\right) \boldsymbol{n}^{-} .
\end{aligned}
$$


This shows the interest of an accurate computation of the jump of the tangential component of the velocity field: $v^{+} \cdot t^{+}+v^{-} \cdot t^{-}$.

Another quantity of physical interest is the circulation around the sail, which we denote $\gamma(\mathcal{S})$ :

$$
\gamma(\mathcal{S}):=-\int_{\mathcal{S}^{+}} \boldsymbol{v}^{+} \cdot \boldsymbol{t}^{+} \mathrm{d} s-\int_{\mathcal{S}^{-}} \boldsymbol{v}^{-} \cdot \boldsymbol{t}^{-} \mathrm{d} s .
$$

Since the domain $\Omega$ is not simply connected, problem (1.1)-(1.2) with boundary conditions (1.4)-(1.5) has a one-parameter family of solutions. In fact, it will be shown below that there exists a unique solution for each arbitrarily given value of the circulation $\gamma(\mathcal{S}) \in \mathbb{R}$. Thus, in order to have uniqueness or, in other words, in order to determine which solution corresponds to the physical flow, we need more information. This is achieved by the so-called Kutta-Joukowski condition which says that the physical flow is not singular at the trailing point $\boldsymbol{x}_{\mathrm{T}}$. Roughly speaking, this leads to the continuity of the velocity at this point and, since $\boldsymbol{v}^{ \pm} \cdot \boldsymbol{n}^{ \pm}=0$ on the sail, it means that taking limit for $\boldsymbol{x} \in \mathcal{S}$,

$$
\lim _{\boldsymbol{x} \rightarrow \boldsymbol{x}_{\mathrm{T}}} \boldsymbol{v}^{+}(\boldsymbol{x}) \cdot \boldsymbol{t}^{+}(\boldsymbol{x})=\lim _{\boldsymbol{x} \rightarrow \boldsymbol{x}_{\mathrm{T}}} \boldsymbol{v}^{-}(\boldsymbol{x}) \cdot \boldsymbol{t}^{+}(\boldsymbol{x}) .
$$

In the next subsection we will give an analytical formulation of the above Kutta-Joukowski condition. More specifically, we will introduce an integral equation to be satisfied by the physical solution, which will allow us to determine the right value of the circulation $\gamma(\mathcal{S})$.

Meanwhile, let us introduce a stream function. Since the velocity $\boldsymbol{v}$ is a solenoidal field, $\boldsymbol{v} \cdot \boldsymbol{n}=0$ on $\mathcal{S}$ and

$$
\int_{\Gamma} \boldsymbol{v} \cdot \boldsymbol{n} \mathrm{d} s=\int_{\Gamma} \boldsymbol{v}_{\infty} \cdot \boldsymbol{n} \mathrm{d} s=0,
$$

we know that there exists a scalar function $\psi$ defined in $\Omega$ such that $\boldsymbol{v}=\operatorname{curl} \psi$, where

$$
\operatorname{curl} \psi:=\frac{\partial \psi}{\partial x_{2}} \boldsymbol{e}_{1}-\frac{\partial \psi}{\partial x_{1}} \boldsymbol{e}_{2} .
$$

From the irrotationality condition we deduce that $\psi$ must be harmonic, i.e.,

$$
\Delta \psi=0
$$

Moreover, boundary condition (1.4) yields

$$
\psi=c \quad \text { on } \mathcal{S},
$$

for some unknown constant $c$. Finally, boundary condition (1.5) becomes

$$
\frac{\partial \psi}{\partial \boldsymbol{t}}=\boldsymbol{v}_{\infty} \cdot \boldsymbol{n} \quad \text { on } \Gamma
$$

which yields, by integration, the Dirichlet condition

$$
\psi=g \quad \text { on } \Gamma
$$

where, for any $\boldsymbol{x} \in \Gamma, g(\boldsymbol{x})$ is the integral of $\boldsymbol{v}_{\infty} \cdot \boldsymbol{n}$ along $\Gamma$ from $\boldsymbol{a}$ to $\boldsymbol{x}$, i.e.,

$$
g(\boldsymbol{x}):=\int_{\Gamma(\boldsymbol{x})} \boldsymbol{v}_{\infty} \cdot \boldsymbol{n} \mathrm{d} s,
$$

$\boldsymbol{a}$ being a chosen point on $\Gamma$ and $\Gamma(\boldsymbol{x})$ the counterclockwise path from $\boldsymbol{a}$ to $\boldsymbol{x}$ along $\Gamma$. Since $\Gamma$ is polygonal, $\boldsymbol{v}_{\infty} \cdot \boldsymbol{n}$ is constant on each edge of $\Gamma$ and hence $g$ is linear on each edge. Moreover it is a continuous function, even at the point $\boldsymbol{a}$ because of (1.8). As a consequence, $g \in \mathrm{H}^{1}(\Gamma)$. 


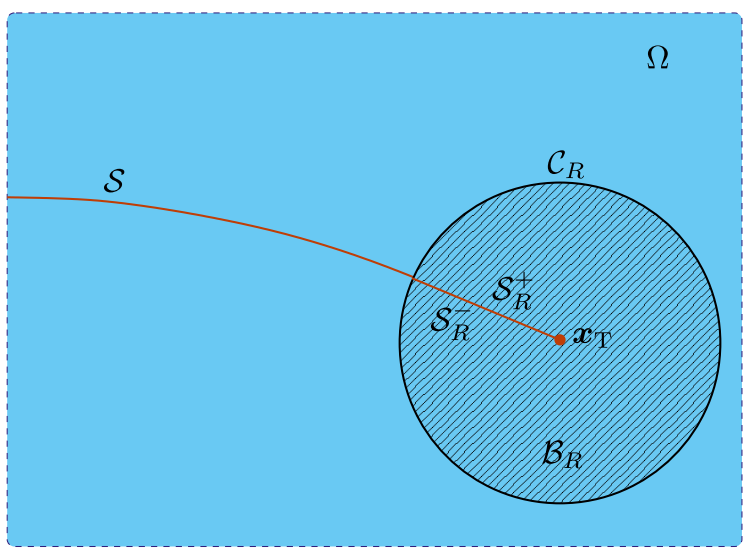

Figure 2. Ball around the trailing edge to impose the Kutta-Joukowski condition.

For each constant $c \in \mathbb{R}$, problem (1.9)-(1.11) has a unique solution in $\mathrm{H}^{1}(\Omega)$. Furthermore, it is the unique solution of the following weak problem:

Problem 1.1. Find $\psi \in \mathrm{H}^{1}(\Omega)$ such that $\psi=g$ on $\Gamma, \psi=c$ on $\mathcal{S}$ and

$$
\int_{\Omega} \operatorname{grad} \psi \cdot \operatorname{grad} z \mathrm{~d} \boldsymbol{x}=0 \quad \forall z \in \mathrm{H}_{0}^{1}(\Omega) .
$$

Let us recall that $\partial \Omega=\Gamma \cup \mathcal{S}$ and, hence, functions in $\mathrm{H}_{0}^{1}(\Omega)$ have a vanishing trace on $\mathcal{S}$ as well as on $\Gamma$.

For each value of $c$, if $\psi$ is the solution of Problem 1.1, then $\boldsymbol{v}=\operatorname{curl} \psi$ satisfies (1.1)-(1.2), with boundary conditions (1.4)-(1.5). In principle, for an arbitrary value of the constant $c$, the solution $\psi$ has two singularities at points $\boldsymbol{x}_{\mathrm{L}}$ and $\boldsymbol{x}_{\mathrm{T}}$. However, the physical solution corresponds to the case where the singularity at the trailing point $\boldsymbol{x}_{\mathrm{T}}$ is removed. This is the classical Kutta-Joukowski condition for which we will give a precise mathematical formulation in what follows.

\subsection{The Kutta-Joukowski condition}

As stated above, for an arbitrary value of the constant $c$, in general the solution of Problem 1.1 becomes singular at the two end points of the sail profile. However, the Kutta-Joukowski condition establishes that the physical motion is not singular at the trailing point. In principle, this condition does not look suitable from the computational point of view. This is why, following the ideas from Ciavaldini et al. [3], we will give an integral condition that guarantees the previous regularity.

For any $r>0$, let $\mathcal{B}_{r}$ denote the ball of radius $r$ centered at $\boldsymbol{x}_{\mathrm{T}}, \mathcal{C}_{r}$ its boundary, $\mathcal{S}_{r}:=\mathcal{S} \cap \mathcal{B}_{r}$ and $\tilde{\mathcal{B}}_{r}:=\mathcal{B}_{r} \backslash \mathcal{S}_{r}$. Let $R>0$ be sufficiently small so that $\mathcal{B}_{R} \subset \subset \Omega$ and $\boldsymbol{x}_{\mathrm{L}} \notin \overline{\mathcal{B}}_{R}$ (see Fig. 2). For the analysis we will assume that $\mathcal{S}_{R}$ is a straight segment. Notice that not the whole sail, but only its piece closest to the trailing point is assumed to be straight. Moreover, the computational experiments show that the integral condition we are going to derive also works although this assumption does not hold.

Let us introduce $(r, \theta), r>0, \theta \in[-\pi, \pi]$ a polar coordinate system centered at the trailing point and such that the segment $\mathcal{S}_{R}$ is contained in the straight half-line $\theta= \pm \pi$. When needed, we will distinguish the segments corresponding to $\theta=\pi$ and $\theta=-\pi$ by introducing the notations $\mathcal{S}_{R}^{+}$and $\mathcal{S}_{R}^{-}$, respectively (see Fig. 2).

Consider the function $\xi(r, \theta):=r^{1 / 2} \cos \frac{\theta}{2}$. Notice that $\xi$ satisfies

$$
\begin{array}{rlrl}
\Delta \xi & =0 & & \text { in } \tilde{\mathcal{B}}_{R}, \\
\xi=0 & & \text { on } \mathcal{S}_{R} .
\end{array}
$$


An easy computation shows that $\xi \in \mathrm{W}^{1, p}\left(\tilde{\mathcal{B}}_{R}\right)$ for $1 \leq p<4$, but $\xi \notin \mathrm{W}^{1,4}\left(\tilde{\mathcal{B}}_{R}\right)$. According to [8], the solution of Problem 1.1 can be decomposed in a neighborhood of $\boldsymbol{x}_{\mathrm{T}}$ as the sum of a singular and a regular part. The former is a multiple of $\xi$, whereas the latter is smoother than $H^{2}\left(\tilde{\mathcal{B}}_{R}\right)$. More precisely, there exists $\alpha \in \mathbb{R}$ and $\tilde{\psi} \in \mathrm{W}^{2, p}\left(\tilde{\mathcal{B}}_{R}\right)$ for all $p \in[1,4)$, such that the solution of Problem 1.1 satisfies

$$
\psi=\alpha \xi+\tilde{\psi} \quad \text { in } \tilde{\mathcal{B}}_{R}
$$

If $\alpha=0$, then $\psi=\tilde{\psi} \in \mathrm{W}^{2, p}\left(\tilde{\mathcal{B}}_{R}\right)$ for some $p>2$ and, from the Sobolev embedding theorem, $\boldsymbol{v}=\operatorname{curl} \psi$ is continuous in the closure of $\tilde{\mathcal{B}}_{R}$. This closure must be understood by taking $\mathcal{S}_{R}^{+}$and $\mathcal{S}_{R}^{-}$as different parts of the boundary of $\tilde{\mathcal{B}}_{R}$, with the trailing point $\boldsymbol{x}_{\mathrm{T}}$ being the only common point. Therefore, although $\boldsymbol{v}$ may have a non vanishing jump on $\mathcal{S}$, it has to be continuous at $\boldsymbol{x}_{\mathrm{T}}$. Instead, if $\alpha \neq 0$, this cannot happen. In fact, straightforward computations show that $|\operatorname{curl} \xi|=\frac{1}{2} r^{-1 / 2}$ and, consequently, for $\alpha \neq 0$, curl $\psi$ is not even bounded at $\boldsymbol{x}_{\mathrm{T}}$.

Let $w_{0}$ be defined by

$$
w_{0}(r, \theta):=\left(r^{-1 / 2}-\frac{r^{1 / 2}}{R}\right) \cos \frac{\theta}{2} .
$$

Then, the function $w_{0}$ belongs to $\mathrm{W}^{1, q}\left(\mathcal{B}_{R}\right)$ for all $q \in\left[1, \frac{4}{3}\right)$ and satisfies

$$
\begin{aligned}
\Delta w_{0}=0 & \text { in } \tilde{\mathcal{B}}_{R}, \\
w_{0}=0 & \text { on } \partial \tilde{\mathcal{B}}_{R}=\mathcal{C}_{R} \cup \mathcal{S}_{R}^{ \pm} .
\end{aligned}
$$

The regularity condition can now be characterized by means of an orthogonality relation. In the proof of the following theorem and thereafter $C$ will denote a generic constant not necessarily the same at each occurrence but always independent of the particular functions involved and, in the forthcoming sections, also independent of the mesh-size parameters.

Theorem 1.2. The solution of Problem 1.1 satisfies $\psi \in \mathrm{W}^{2, p}\left(\tilde{\mathcal{B}}_{R}\right)$ for all $p \in[1,4)$ if and only if

$$
\int_{\mathcal{C}_{R}}(\psi-c) \frac{\partial w_{0}}{\partial \boldsymbol{n}} \mathrm{d} s=0
$$

Proof. First we prove that the regularity condition implies (1.17). Let $\psi \in \mathrm{W}^{2, p}\left(\tilde{\mathcal{B}}_{R}\right)$ for all $p \in[1,4)$. By using a Green's formula, (1.9) and (1.16), we have

$$
\lim _{\epsilon \rightarrow 0} \int_{\tilde{\mathcal{B}}_{R} \backslash \tilde{\mathcal{B}}_{\epsilon}} \operatorname{grad} \psi \cdot \operatorname{grad} w_{0} \mathrm{~d} \boldsymbol{x}=-\lim _{\epsilon \rightarrow 0} \int_{\tilde{\mathcal{B}}_{R} \backslash \tilde{\mathcal{B}}_{\epsilon}} \Delta \psi w_{0} \mathrm{~d} \boldsymbol{x}+\lim _{\epsilon \rightarrow 0} \int_{\partial\left(\tilde{\mathcal{B}}_{R} \backslash \tilde{\mathcal{B}}_{\epsilon}\right)} \frac{\partial \psi}{\partial \boldsymbol{n}} w_{0} \mathrm{~d} s=\lim _{\epsilon \rightarrow 0} \int_{\mathcal{C}_{\epsilon}} \frac{\partial \psi}{\partial \boldsymbol{n}} w_{0} \mathrm{~d} s
$$

whereas, from the explicit expression of $w_{0}$ and the Sobolev embedding theorem, for any $p \in(2,4)$ we have

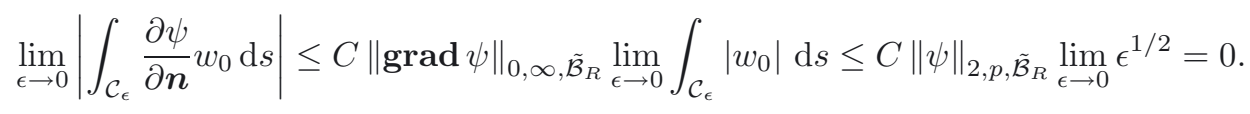

On the other hand, using a Green's formula again, (1.15) and (1.10),

$$
\begin{aligned}
\lim _{\epsilon \rightarrow 0} \int_{\tilde{\mathcal{B}}_{R} \backslash \tilde{\mathcal{B}}_{\epsilon}} \operatorname{grad} \psi \cdot \operatorname{grad} w_{0} \mathrm{~d} \boldsymbol{x} & =-\lim _{\epsilon \rightarrow 0} \int_{\tilde{\mathcal{B}}_{R} \backslash \tilde{\mathcal{B}}_{\epsilon}}(\psi-c) \Delta w_{0} \mathrm{~d} \boldsymbol{x}+\lim _{\epsilon \rightarrow 0} \int_{\partial\left(\tilde{\mathcal{B}}_{R} \backslash \tilde{\mathcal{B}}_{\epsilon}\right)}(\psi-c) \frac{\partial w_{0}}{\partial \boldsymbol{n}} \mathrm{d} s \\
& =\lim _{\epsilon \rightarrow 0} \int_{\mathcal{C}_{R} \cup \mathcal{C}_{\epsilon}}(\psi-c) \frac{\partial w_{0}}{\partial \boldsymbol{n}} \mathrm{d} s
\end{aligned}
$$


Therefore,

Finally, for $\boldsymbol{x} \in \mathcal{C}_{\epsilon}$,

$$
\int_{\mathcal{C}_{R}} \frac{\partial w_{0}}{\partial \boldsymbol{n}}(\psi-c) \mathrm{d} s=-\lim _{\epsilon \rightarrow 0} \int_{\mathcal{C}_{\epsilon}} \frac{\partial w_{0}}{\partial \boldsymbol{n}}(\psi-c) \mathrm{d} s
$$

$$
|\psi(\boldsymbol{x})-c|=\left|\psi(\boldsymbol{x})-\psi\left(\boldsymbol{x}_{\mathrm{T}}\right)\right| \leq\|\operatorname{grad} \psi\|_{0, \infty, \tilde{\mathcal{B}}_{R}}\left|\boldsymbol{x}-\boldsymbol{x}_{\mathrm{T}}\right| \leq C\|\psi\|_{2, p, \tilde{\mathcal{B}}_{R}} \epsilon
$$

for any $p \in(2,4)$. Hence, from explicit computations with $w_{0}$ we obtain

$$
\left|\lim _{\epsilon \rightarrow 0} \int_{\mathcal{C}_{\epsilon}} \frac{\partial w_{0}}{\partial \boldsymbol{n}}(\psi-c) \mathrm{d} s\right| \leq C\|\psi\|_{2, p, \tilde{\mathcal{B}}_{R}} \lim _{\epsilon \rightarrow 0} \epsilon \int_{\mathcal{C}_{\epsilon}}\left|\frac{\partial w_{0}}{\partial \boldsymbol{n}}\right| \mathrm{d} s \leq C\|\psi\|_{2, p, \tilde{\mathcal{B}}_{R}} \lim _{\epsilon \rightarrow 0} \epsilon^{1 / 2}=0,
$$

which allows us to conclude (1.17).

Conversely, we have to prove that if $\alpha \neq 0$ in (1.13), then (1.17) does not hold. First, we notice that $\tilde{\psi}=\psi-\alpha \xi$ is harmonic in $\tilde{\mathcal{B}}_{R}$, satisfies the same boundary condition as $\psi$ on $\mathcal{S}^{ \pm}$and belongs to $\mathrm{W}^{2, p}\left(\tilde{\mathcal{B}}_{R}\right)$ for all $p \in[1,4)$. Thus, we have just proved that

$$
\int_{\mathcal{C}_{R}}(\psi-\alpha \xi-c) \frac{\partial w_{0}}{\partial \boldsymbol{n}} \mathrm{d} s=0
$$

On the other hand, explicit computations yield

$$
\int_{\mathcal{C}_{R}} \xi \frac{\partial w_{0}}{\partial \boldsymbol{n}} \mathrm{d} s=-\pi
$$

Hence, for all $\alpha \neq 0, \int_{\mathcal{C}_{R}}(\psi-c) \frac{\partial w_{0}}{\partial \boldsymbol{n}} \mathrm{d} s=\alpha \int_{\mathcal{C}_{R}} \xi \frac{\partial w_{0}}{\partial \boldsymbol{n}} \mathrm{d} s \neq 0$ and we conclude the proof.

As a consequence of the above theorem we have that the stream function leading to the physical solution of Problem 1.1 can be obtained by solving the following problem:

Problem 1.3. Find $\psi \in \mathrm{H}^{1}(\Omega)$ and $c \in \mathbb{R}$ such that $\psi=g$ on $\Gamma$ and

$$
\begin{array}{rlrl}
\int_{\Omega} \operatorname{grad} \psi \cdot \operatorname{grad} z \mathrm{~d} \boldsymbol{x} & =0 & & \forall z \in \mathrm{H}_{0}^{1}(\Omega), \\
\psi & =c \quad \text { on } \mathcal{S}, \\
\int_{\mathcal{C}_{R}}(\psi-c) \frac{\partial w_{0}}{\partial \boldsymbol{n}} \mathrm{d} s & =0 . & &
\end{array}
$$

\section{A Fictitious DOMAin FORMUlation}

In practical applications the sail shape is unknown, so we are led to solve a typical fluid-structure interaction problem. A standard method for such problem consists in starting from an initial shape, which is updated along an iterative process involving flow problems for given sail shapes and structural problems for given aerodynamic forces.

In order to avoid remeshing at each iteration as the sail shape changes, we propose a fictitious domain method in which the presence of the sail in the flow domain is taken into account by introducing a Lagrange multiplier. More specifically, the Dirichlet boundary condition on the sail, $\psi=c$, is imposed in a weak form by introducing a Lagrange multiplier and, accordingly, we eliminate the constraint on the test function $z=0$ on $\mathcal{S}$ in (1.18).

With this purpose, we endow $\mathrm{H}_{0}^{1}(\mathcal{R})$ (recall that $\mathcal{R}:=\Omega \cup \mathcal{S}$ ) with the norm

$$
\|z\|_{\mathrm{H}_{0}^{1}(\mathcal{R})}:=\left(\int_{\mathcal{R}}|\operatorname{grad} z|^{2} \mathrm{~d} \boldsymbol{x}\right)^{1 / 2}
$$


which is equivalent to the standard $\mathrm{H}^{1}(\mathcal{R})$ norm. Also, we identify $\mathrm{H}^{1 / 2}(\mathcal{S})$ with the space of traces on $\mathcal{S}$ of functions in $\mathrm{H}_{0}^{1}(\mathcal{R})$, endowed with the norm

$$
\|\zeta\|_{\mathrm{H}^{1 / 2}(\mathcal{S})}:=\inf \left\{\|z\|_{\mathrm{H}_{0}^{1}(\mathcal{R})}, z \in \mathrm{H}_{0}^{1}(\mathcal{R}):\left.z\right|_{\mathcal{S}}=\zeta\right\}
$$

which in its turn is equivalent to the intrinsic norm of this space, as defined for instance in [6, Sect. I.1, (1.9)]. Finally, we denote by $\langle\cdot, \cdot\rangle_{\mathcal{S}}$ the duality pairing between $\mathrm{H}^{1 / 2}(\mathcal{S})^{\prime}$ and $\mathrm{H}^{1 / 2}(\mathcal{S})$. Hence, the problem to be solved is the following:

Problem 2.1. Find $\psi \in \mathrm{H}^{1}(\mathcal{R}), \lambda \in \mathrm{H}^{1 / 2}(\mathcal{S})^{\prime}$ and $c \in \mathbb{R}$ such that $\psi=g$ on $\Gamma$ and

$$
\begin{aligned}
\int_{\mathcal{R}} \operatorname{grad} \psi \cdot \operatorname{grad} z \mathrm{~d} \boldsymbol{x}+\langle\lambda, z\rangle_{\mathcal{S}} & =0 \quad \forall z \in \mathrm{H}_{0}^{1}(\mathcal{R}), \\
\langle\mu, \psi-c\rangle_{\mathcal{S}} & =0 \quad \forall \mu \in \mathrm{H}^{1 / 2}(\mathcal{S})^{\prime}, \\
\int_{\mathcal{C}_{R}}(\psi-c) \frac{\partial w_{0}}{\partial \boldsymbol{n}} \mathrm{d} s & =0 .
\end{aligned}
$$

Problems 1.3 and 2.1 are equivalent. In fact, if $(\psi, \lambda, c)$ is a solution of the latter, then clearly $(\psi, c)$ solves the former. Conversely, let $(\psi, c)$ be a solution of Problem 1.3 and let

$$
\begin{aligned}
\lambda: \mathrm{H}^{1 / 2}(\mathcal{S}) & \rightarrow \mathbb{R} \\
\zeta & \mapsto-\int_{\Omega} \operatorname{grad} \psi \cdot \operatorname{grad} z \mathrm{~d} \boldsymbol{x},
\end{aligned}
$$

where $z$ is any function in $\mathrm{H}_{0}^{1}(\mathcal{R})$ such that $\left.z\right|_{\mathcal{S}}=\zeta$. The functional $\lambda$ is well defined since, if $\hat{z} \in \mathrm{H}_{0}^{1}(\mathcal{R})$ also satisfies $\left.\hat{z}\right|_{\mathcal{S}}=\zeta$, then $\hat{z}-z \in \mathrm{H}_{0}^{1}(\Omega)$ and, hence, $\int_{\Omega} \operatorname{grad} \psi \cdot \operatorname{grad} \hat{z} \mathrm{~d} \boldsymbol{x}=\int_{\Omega} \operatorname{grad} \psi \cdot \operatorname{grad} z \mathrm{~d} \boldsymbol{x}$, because of (1.18). Moreover, $\lambda \in \mathrm{H}^{1 / 2}(\mathcal{S})^{\prime}$ since $\left|\langle\lambda, \zeta\rangle_{\mathcal{S}}\right| \leq\|\operatorname{grad} \psi\|_{\mathrm{L}^{2}(\Omega)^{2}}\|z\|_{\mathrm{H}_{0}^{1}(\mathcal{R})}$ for all $z \in \mathrm{H}_{0}^{1}(\mathcal{R})$ such that $\left.z\right|_{\mathcal{S}}=\zeta$ and, hence, $\|\lambda\|_{\mathrm{H}^{1 / 2}(\mathcal{S})^{\prime}} \leq\|\operatorname{grad} \psi\|_{\mathrm{L}^{2}(\Omega)^{2}}$. Therefore, $(\psi, \lambda, c)$ is a solution of Problem 2.1.

Remark 2.2. Consider the following mapping:

$$
\begin{aligned}
\mathrm{H}^{1 / 2}(\mathcal{S})^{\prime} & \rightarrow \mathrm{H}_{0}^{1}(\mathcal{R}), \\
\mu & \mapsto \phi_{\mu}
\end{aligned}
$$

where $\phi_{\mu} \in \mathrm{H}_{0}^{1}(\mathcal{R})$ is the unique solution of the problem

$$
\int_{\mathcal{R}} \operatorname{grad} \phi_{\mu} \cdot \operatorname{grad} z \mathrm{~d} \boldsymbol{x}=\langle\mu, z\rangle_{\mathcal{S}} \quad \forall z \in \mathrm{H}_{0}^{1}(\mathcal{R}) .
$$

It is simple to show that $\|\mu\|_{\mathrm{H}^{1 / 2}(\mathcal{S})^{\prime}}=\left\|\phi_{\mu}\right\|_{\mathrm{H}_{0}^{1}(\mathcal{R})}$. In fact, first notice that

$$
\left\|\phi_{\mu}\right\|_{\mathrm{H}_{0}^{1}(\mathcal{R})}^{2}=\int_{\mathcal{R}} \operatorname{grad} \phi_{\mu} \cdot \operatorname{grad} \phi_{\mu} \mathrm{d} \boldsymbol{x}=\left\langle\mu, \phi_{\mu}\right\rangle_{\mathcal{S}} \leq\|\mu\|_{\mathrm{H}^{1 / 2}(\mathcal{S})^{\prime}}\left\|\left.\phi_{\mu}\right|_{\mathcal{S}}\right\|_{\mathrm{H}^{1 / 2}(\mathcal{S})} \leq\|\mu\|_{\mathrm{H}^{1 / 2}(\mathcal{S})^{\prime}}\left\|\phi_{\mu}\right\|_{\mathrm{H}_{0}^{1}(\mathcal{R})}
$$

and, hence, $\left\|\phi_{\mu}\right\|_{\mathrm{H}_{0}^{1}(\mathcal{R})} \leq\|\mu\|_{\mathrm{H}^{1 / 2}(\mathcal{S})^{\prime}}$. On the other hand,

$$
\|\mu\|_{\mathrm{H}^{1 / 2}(\mathcal{S})^{\prime}}:=\sup _{\zeta \in \mathrm{H}^{1 / 2}(\mathcal{S})} \frac{\langle\mu, \zeta\rangle_{\mathcal{S}}}{\|\zeta\|_{\mathrm{H}^{1 / 2}(\mathcal{S})}}=\sup _{z \in \mathrm{H}_{0}^{1}(\mathcal{R})} \frac{\langle\mu, z\rangle_{\mathcal{S}}}{\|z\|_{\mathrm{H}_{0}^{1}(\mathcal{R})}},
$$

and, hence,

$$
\|\mu\|_{\mathrm{H}^{1 / 2}(\mathcal{S})^{\prime}}=\sup _{z \in \mathrm{H}_{0}^{1}(\mathcal{R})} \frac{\int_{\mathcal{R}} \operatorname{grad} \phi_{\mu} \cdot \operatorname{grad} z \mathrm{~d} \boldsymbol{x}}{\|z\|_{\mathrm{H}_{0}^{1}(\mathcal{R})}} \leq\left\|\phi_{\mu}\right\|_{\mathrm{H}_{0}^{1}(\mathcal{R})} .
$$


Thus, we conclude that $\|\mu\|_{\mathrm{H}^{1 / 2}(\mathcal{S})^{\prime}}=\left\|\phi_{\mu}\right\|_{\mathrm{H}_{0}^{1}(\mathcal{R})}$. This will be used in Section 4 to compute the $\mathrm{H}^{1 / 2}(\mathcal{S})^{\prime}$-norm.

Remark 2.3. The Lagrange multiplier $\lambda$ can be used to compute the local force exerted by the flow on the sail by means of (1.6), as follows:

$$
\boldsymbol{f} \approx \rho v_{\infty} \lambda \boldsymbol{n}^{-} .
$$

Indeed, testing (2.1) with $z \in \mathcal{D}(\Omega)$, we have that $\psi$ is harmonic in $\Omega$. Therefore, by using a Green's formula in (2.1), we deduce, for all $z \in \mathrm{H}_{0}^{1}(\mathcal{R})$,

$$
\langle\lambda, z\rangle_{\mathcal{S}}=-\int_{\mathcal{S}^{+}} \frac{\partial \psi^{+}}{\partial \boldsymbol{n}^{+}} z \mathrm{~d} s-\int_{\mathcal{S}^{-}} \frac{\partial \psi^{-}}{\partial \boldsymbol{n}^{-}} z \mathrm{~d} s=-\int_{\mathcal{S}}\left(\frac{\partial \psi^{+}}{\partial \boldsymbol{n}^{+}}+\frac{\partial \psi^{-}}{\partial \boldsymbol{n}^{-}}\right) z \mathrm{~d} s
$$

as long as the integrals above make sense. Thus, for $\boldsymbol{v}=\operatorname{curl} \psi$,

$$
\lambda=-\left(\frac{\partial \psi^{+}}{\partial \boldsymbol{n}^{+}}+\frac{\partial \psi^{-}}{\partial \boldsymbol{n}^{-}}\right)=\boldsymbol{v}^{+} \cdot \boldsymbol{t}^{+}+\boldsymbol{v}^{-} \cdot \boldsymbol{t}^{-}
$$

which together with (1.6) yield (2.6).

Remark 2.4. The term above corresponds to the jump of the normal derivative of $\psi$ across the sail:

$$
\llbracket \frac{\partial \psi}{\partial \boldsymbol{n}} \rrbracket_{\mathcal{S}}:=\frac{\partial \psi^{+}}{\partial \boldsymbol{n}^{+}}+\frac{\partial \psi^{-}}{\partial \boldsymbol{n}^{-}}
$$

\subsection{Existence and uniqueness of solution to Problem 2.1}

In order to prove the existence of a solution to Problem 2.1 we introduce two auxiliary problems. With this aim, let $\omega \subset \subset \mathcal{R}$ be an open set such that $\mathcal{S} \cup \mathcal{C}_{R} \subset \omega$ and let $\psi_{g} \in \mathrm{H}^{1}(\mathcal{R})$ be a function such that $\operatorname{supp}\left(\psi_{g}\right) \subset \overline{\mathcal{R}} \backslash \bar{\omega}$ and $\left.\psi_{g}\right|_{\Gamma}=g$. Consider the two following auxiliary mixed problems:

- Find $\psi_{1} \in \mathrm{H}_{0}^{1}(\mathcal{R})$ and $\lambda_{1} \in \mathrm{H}^{1 / 2}(\mathcal{S})^{\prime}$ such that

$$
\begin{aligned}
\int_{\mathcal{R}} \operatorname{grad} \psi_{1} \cdot \operatorname{grad} z \mathrm{~d} \boldsymbol{x}+\left\langle\lambda_{1}, z\right\rangle_{\mathcal{S}}=0 & \forall z \in \mathrm{H}_{0}^{1}(\mathcal{R}), \\
\left\langle\mu, \psi_{1}\right\rangle_{\mathcal{S}}=\langle\mu, 1\rangle_{\mathcal{S}} & \forall \mu \in \mathrm{H}^{1 / 2}(\mathcal{S})^{\prime} .
\end{aligned}
$$

- Find $\psi_{2} \in \mathrm{H}_{0}^{1}(\mathcal{R})$ and $\lambda_{2} \in \mathrm{H}^{1 / 2}(\mathcal{S})^{\prime}$ such that

$$
\begin{array}{rr}
\int_{\mathcal{R}} \operatorname{grad} \psi_{2} \cdot \operatorname{grad} z \mathrm{~d} \boldsymbol{x}+\left\langle\lambda_{2}, z\right\rangle_{\mathcal{S}}=-\int_{\mathcal{R}} \operatorname{grad} \psi_{g} \cdot \operatorname{grad} z \mathrm{~d} \boldsymbol{x} & \forall z \in \mathrm{H}_{0}^{1}(\mathcal{R}), \\
\left\langle\mu, \psi_{2}\right\rangle_{\mathcal{S}}=0 & \forall \mu \in \mathrm{H}^{1 / 2}(\mathcal{S})^{\prime} .
\end{array}
$$

The existence and uniqueness of solution to these two problems follows directly from the standard theory of mixed problems (see, for instance, [2] or [6]) by virtue of the $\mathrm{H}_{0}^{1}(\mathcal{R})$-ellipticity of the bilinear form $(\psi, z) \mapsto$ $\int_{\mathcal{R}} \operatorname{grad} \psi \cdot \operatorname{grad} z \mathrm{~d} \boldsymbol{x}$ and the inf-sup condition arising from (2.5).

Now we can prove the following result:

Theorem 2.5. Let us assume that the solution of problem (2.8)-(2.9) is such that

$$
\int_{\mathcal{C}_{R}}\left(\psi_{1}-1\right) \frac{\partial w_{0}}{\partial \boldsymbol{n}} \mathrm{d} s \neq 0
$$

Then, there exists a unique solution to Problem 2.1. 
Proof. Existence: We observe that $\left\langle\mu, \psi_{g}\right\rangle_{\mathcal{S}}=0$ for all $\mu \in \mathrm{H}^{1 / 2}(\mathcal{S})^{\prime}$, because $\operatorname{supp}\left(\psi_{g}\right) \cap \mathcal{S}=\emptyset$, and then

$$
\begin{aligned}
\int_{\mathcal{R}} \operatorname{grad}\left(\psi_{2}+\psi_{g}\right) \cdot \operatorname{grad} z \mathrm{~d} \boldsymbol{x}+\left\langle\lambda_{2}, z\right\rangle_{\mathcal{S}} & =0 \quad \forall z \in \mathrm{H}_{0}^{1}(\mathcal{R}), \\
\left\langle\mu, \psi_{2}+\psi_{g}\right\rangle_{\mathcal{S}} & =0 \quad \forall \mu \in \mathrm{H}^{1 / 2}(\mathcal{S})^{\prime} .
\end{aligned}
$$

Let the constant $c$ be defined as follows:

$$
c:=-\frac{\int_{\mathcal{C}_{R}} \psi_{2} \frac{\partial w_{0}}{\partial \boldsymbol{n}} \mathrm{d} s}{\int_{\mathcal{C}_{R}}\left(\psi_{1}-1\right) \frac{\partial w_{0}}{\partial \boldsymbol{n}} \mathrm{d} s} .
$$

Then, it is immediate to see that defining

$$
\psi:=c \psi_{1}+\psi_{2}+\psi_{g}
$$

and

$$
\lambda:=c \lambda_{1}+\lambda_{2}
$$

$(\psi, \lambda, c)$ is a solution to Problem 2.1 .

Uniqueness: Let $(\psi, \lambda, c)$ and $(\hat{\psi}, \hat{\lambda}, \hat{c})$ be two solutions to Problem 2.1. Then, the difference $\left(\psi_{d}, \lambda_{d}, c_{d}\right):=$ $(\psi, \lambda, c)-(\hat{\psi}, \hat{\lambda}, \hat{c})$ satisfies $\psi_{d}=0$ on $\Gamma$ and

$$
\begin{aligned}
& \int_{\mathcal{R}} \operatorname{grad} \psi_{d} \cdot \operatorname{grad} z \mathrm{~d} \boldsymbol{x}+\left\langle\lambda_{d}, z\right\rangle_{\mathcal{S}}=0 \quad \forall z \in \mathrm{H}_{0}^{1}(\mathcal{R}), \\
& \left\langle\mu, \psi_{d}-c_{d}\right\rangle_{\mathcal{S}}=0 \quad \forall \mu \in \mathrm{H}^{1 / 2}(\mathcal{S})^{\prime}, \\
& \int_{\mathcal{C}_{R}}\left(\psi_{d}-c_{d}\right) \frac{\partial w_{0}}{\partial \boldsymbol{n}} \mathrm{d} s=0 \text {. }
\end{aligned}
$$

From the uniqueness of the solution of (2.8)-(2.9), we deduce $\psi_{d}=c_{d} \psi_{1}$ and $\lambda_{d}=c_{d} \lambda_{1}$. Hence, (2.16) implies

$$
c_{d} \int_{\mathcal{C}_{R}}\left(\psi_{1}-1\right) \frac{\partial w_{0}}{\partial \boldsymbol{n}} \mathrm{d} s=0,
$$

from which it follows that $c_{d}=0$, because of assumption (2.12). Then, $\psi_{d}=0$ and $\lambda_{d}=0$.

Remark 2.6. We have used assumption (2.12) to derive the existence and uniqueness of solution to Problem 2.1 and we will use it again throughout the next section. This assumption is quite plausible. In fact, problem (2.8)(2.9) is equivalent to Problem 1.1 with $g=0$ and $c=1$, which is a weak formulation of the following one:

$$
\begin{aligned}
\Delta \psi_{1}=0 & \text { in } \Omega \\
\psi_{1}=0 & \text { on } \Gamma \\
\psi_{1}=1 & \text { on } \mathcal{S} .
\end{aligned}
$$

Thus, as a consequence of Theorem 1.2, assumption (2.12) holds true unless $\psi_{1}$ were specially smooth in a neighborhood of the trailing point $\boldsymbol{x}_{\mathrm{T}}$ (i.e., $\psi_{1} \in \mathrm{W}^{2, p}\left(\tilde{\mathcal{B}}_{R}\right)$, with $p>2$ ). This never happened in our experiments. 
Remark 2.7. We have stated above that problem (1.1)-(1.2) with boundary conditions (1.4)-(1.5) has a unique solution for each arbitrary value of the circulation $\gamma(\mathcal{S})$. Indeed, we have already shown that, by taking $\boldsymbol{v}=$ $\operatorname{curl} \psi$ and $g$ defined by (1.12), these equations are equivalent to Problem 1.3. In its turn, equations (1.18)-(1.20) from this problem have a unique solution $(\psi, \lambda)$ for each value of the constant $c$. For this solution, the same arguments used in the proof of Theorem 2.5 allow us to write $\lambda=c \lambda_{1}+\lambda_{2}$, with $\left(\psi_{1}, \lambda_{1}\right)$ and $\left(\psi_{2}, \lambda_{2}\right)$ being the solutions of problems (2.8)-(2.9) and (2.10)-(2.11), respectively. Therefore, from (1.7) and (2.7),

$$
-\gamma(\mathcal{S})=\langle\lambda, 1\rangle_{\mathcal{S}}=c\left\langle\lambda_{1}, 1\right\rangle_{\mathcal{S}}+\left\langle\lambda_{2}, 1\right\rangle_{\mathcal{S}}
$$

Since, $\psi_{1} \in \mathrm{H}_{0}^{1}(\mathcal{R})$ and $\psi_{1}=1$ on $\mathcal{S}(c f .(2.9))$, (2.8) yields

$$
\left\langle\lambda_{1}, 1\right\rangle_{\mathcal{S}}=\left\langle\lambda_{1}, \psi_{1}\right\rangle_{\mathcal{S}}=-\int_{\mathcal{R}}\left|\operatorname{grad} \psi_{1}\right|^{2} \mathrm{~d} \boldsymbol{x} \neq 0
$$

so that each value of the circulation corresponds to a unique value of the constant $c$.

\section{NumericAl approximation}

Let $\left\{\mathcal{T}_{h}\right\}$ be a regular family of triangulations of $\mathcal{R} ; h$ denotes as usual the corresponding mesh-size. Let

$$
\mathcal{Z}_{h}:=\left\{z_{h} \in \mathrm{H}^{1}(\mathcal{R}):\left.z_{h}\right|_{T} \in \mathbb{P}_{1} \forall T \in \mathcal{T}_{h}\right\} \quad \text { and } \quad \mathcal{Z}_{h}^{0}:=\mathcal{Z}_{h} \cap \mathrm{H}_{0}^{1}(\mathcal{R})
$$

We assume all the meshes are refinements of a fixed coarse one, $\mathcal{T}_{h_{0}}$, such that $\bar{\omega} \cap \bigcup\left\{T \in \mathcal{T}_{h_{0}}: T \cap \Gamma \neq \emptyset\right\}=\emptyset$. (We recall $\omega$ is the open set introduced at the beginning of Section 2.1, which satisfies $\omega \subset \subset \mathcal{R}$ and $\omega \supset \mathcal{S} \cup \mathcal{C}_{R}$.) Since $g$ is piecewise linear and continuous on $\Gamma$, this allows us to choose, for problem $(2.10)-(2.11), \psi_{g} \in \mathcal{Z}_{h_{0}}$ such that $\left.\psi_{g}\right|_{\Gamma}=g$ and $\left.\psi_{g}\right|_{\bar{\omega}}=0$. Then, as a consequence of the assumption above, $\psi_{g} \in \mathcal{Z}_{h}$ for all meshes $\mathcal{T}_{h}$. This is the reason for such assumption, which, although not actually necessary, makes the proofs that follow a bit simpler.

For each mesh $\mathcal{T}_{h}$, we consider a partition of $\mathcal{S}$ into curved $\operatorname{arcs}, \mathcal{T}_{H}:=\left\{S_{1}, \ldots, S_{N}\right\}$. The mesh parameter is in this case $H:=\max \left\{\operatorname{length}\left(S_{j}\right), j=1, \ldots, N\right\}$. We will use the following finite dimensional subspace of $\mathrm{H}^{1 / 2}(\mathcal{S})^{\prime}$ to approximate the Lagrange multiplier:

$$
\mathcal{M}_{H}:=\left\{\mu_{H} \in \mathrm{L}^{2}(\mathcal{S}):\left.\mu_{H}\right|_{S_{j}} \in \mathbb{P}_{0} \forall S_{j} \in \mathcal{T}_{H}\right\}
$$

We introduce some additional notation and assumptions concerning the meshes. Let

$$
\begin{aligned}
& \mathcal{N}_{h}=\left\{P_{1}, P_{2}, \ldots, P_{M}\right\}: \text { set of inner vertices of } \mathcal{T}_{h}, \\
& \varphi_{i}: \text { nodal basis function, for all } P_{i} \in \mathcal{N}_{h}, \\
& \omega_{i}:=\bigcup\left\{T \in \mathcal{T}_{h}: T \ni P_{i}\right\}: \text { support of } \varphi_{i}, \text { for all } P_{i} \in \mathcal{N}_{h}, \\
& \Omega_{T}:=\bigcup\left\{T^{\prime} \in \mathcal{T}_{h}: T^{\prime} \cap T \neq \emptyset\right\}, \text { for all } T \in \mathcal{T}_{h}, \\
& h_{i}:=\max \left\{\operatorname{diam}(T): T \in \mathcal{T}_{h}, T \ni P_{i}\right\}, \text { for all } P_{i} \in \mathcal{N}_{h}, \\
& \tilde{\Omega}_{j}:=\bigcup\left\{T \in \mathcal{T}_{h}: T \cap S_{j} \neq \emptyset\right\}, \text { for all } S_{j} \in \mathcal{T}_{H}, \\
& \Omega_{j}:=\bigcup\left\{T \in \mathcal{T}_{h}: T \cap \tilde{\Omega}_{j} \neq \emptyset\right\}, \text { for all } S_{j} \in \mathcal{T}_{H} .
\end{aligned}
$$

We assume that each mesh $\mathcal{T}_{h}$ and the corresponding mesh $\mathcal{T}_{H}$ on $\mathcal{S}$ satisfy the following constraints:

Assumption: For each $S_{j} \in \mathcal{T}_{H}$, there exists a vertex $P_{k_{j}} \in \mathcal{N}_{h}$ such that

(i) $\omega_{k_{j}} \cap \mathcal{S} \subset S_{j}$ 
(ii) the sets $\omega_{k_{j}}, j=1, \ldots, N$, are mutually disjoint,

(iii) there exists a constant $\underline{L}>0$ such that $\int_{S_{j}} \varphi_{k_{j}} \mathrm{~d} s \geq \underline{L} h_{k_{j}}$ and

(iv) there exists a constant $\bar{L}>0$ such that length $\left(S_{j}\right) \leq \bar{L} h_{k_{j}}$, for all $S_{j} \in \mathcal{T}_{H}$.

Constants $\bar{L}$ and $\underline{L}$ above are independent of $h$ and $H$. Therefore, as an immediate consequence of assumption (iv), $H \rightarrow 0$ as $h \rightarrow 0$.

Meshes with these properties have been introduced and used in [5], where a thorough analysis of the fictitious domain method to impose a Dirichlet condition is performed. In practice, the assumptions above are fulfilled provided the mesh-size on the sail, $H$, is about two or three times larger than that on the domain, $h$, and the angles at the corners of $\mathcal{S}$ are not too small, the latter in the case that the sail were not a smooth curve. See [5] for further details.

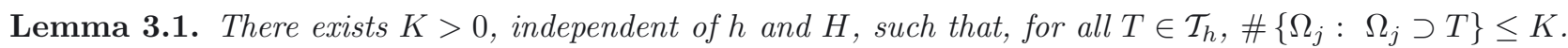

Proof. Let $T \in \mathcal{T}_{h}$. For each $\Omega_{j}$ such that $\Omega_{j} \supset T$, let $P_{k_{j}} \in \mathcal{N}_{h}$ be as in assumption (i). We have

$$
\operatorname{dist}\left(T, P_{k_{j}}\right) \leq \operatorname{dist}\left(T, S_{j}\right)+\operatorname{length}\left(S_{j}\right)+\operatorname{dist}\left(S_{j}, P_{k_{j}}\right) \leq C_{1} h_{k_{j}}+\bar{L} h_{k_{j}}+C_{2} h_{k_{j}},
$$

with $C_{1}$ and $C_{2}$ constants which only depend on the regularity of the mesh and $\bar{L}$ being the constant from assumption (iv). Hence, denoting $C_{3}:=C_{1}+\bar{L}+C_{2}$, there holds

$$
\#\left\{\Omega_{j}: \Omega_{j} \supset T\right\} \leq \#\left\{P_{k_{j}}: \operatorname{dist}\left(P_{k_{j}}, T\right) \leq C_{3} h_{k_{j}}\right\} \leq \#\left\{P_{k} \in \mathcal{N}_{h}: \operatorname{dist}\left(P_{k}, T\right) \leq C_{3} h\right\}
$$

and the lemma follows from the fact that the latter is bounded above by a number that only depends on the regularity of the mesh, as well.

The above assumptions allow proving the existence of a Fortin operator for the auxiliary mixed problems (2.8)-(2.9) and (2.10)-(2.11). From now on, the generic constant $C$ will be always independent of the mesh parameters $h$ and $H$.

Lemma 3.2. There exists a linear operator $\Pi_{h}: \mathrm{H}_{0}^{1}(\mathcal{R}) \rightarrow \mathcal{Z}_{h}^{0}$ such that

$$
\begin{aligned}
\left\|\Pi_{h} z\right\|_{\mathrm{H}_{0}^{1}(\mathcal{R})} \leq C\|z\|_{\mathrm{H}_{0}^{1}(\mathcal{R})} & \forall z \in \mathrm{H}_{0}^{1}(\mathcal{R}), \\
\left\langle\mu_{H}, \Pi_{h} z\right\rangle_{\mathcal{S}}=\left\langle\mu_{H}, z\right\rangle_{\mathcal{S}} & \forall z \in \mathrm{H}_{0}^{1}(\mathcal{R}) \quad \forall \mu_{H} \in \mathcal{M}_{H} .
\end{aligned}
$$

Proof. Let $\mathcal{I}_{h}: \mathrm{H}_{0}^{1}(\mathcal{R}) \rightarrow \mathcal{Z}_{h}$ be a Clément interpolant preserving zero values on $\partial \mathcal{R}$ (see [13]). There holds

$$
\begin{aligned}
\left\|\mathcal{I}_{h} z\right\|_{\mathrm{H}^{1}(T)} \leq C\|z\|_{\mathrm{H}^{1}\left(\Omega_{T}\right)} & \forall z \in \mathrm{H}^{1}(\mathcal{R}), \\
\left\|z-\mathcal{I}_{h} z\right\|_{\mathrm{L}^{2}\left(S_{j}\right)} \leq C h_{k_{j}}^{1 / 2}\|\operatorname{grad} z\|_{\mathrm{L}^{2}\left(\Omega_{k_{j}}\right)} & \forall z \in \mathrm{H}^{1}(\mathcal{R}) .
\end{aligned}
$$

We define $\Pi_{h} z:=\mathcal{I}_{h} z+\sum_{j=1}^{N} c_{j} \varphi_{k_{j}}$, with

$$
c_{j}:=\frac{\int_{S_{j}}\left(z-\mathcal{I}_{h} z\right) \mathrm{d} s}{\int_{S_{j}} \varphi_{k_{j}} \mathrm{~d} s}, \quad j=1, \ldots, N
$$

which are well defined because $\int_{S_{j}} \varphi_{k_{j}} \mathrm{~d} s>0$, as a consequence of assumption (iii). Therefore, by virtue of assumption (i),

$$
\int_{S_{i}} \Pi_{h} z \mathrm{~d} s=\int_{S_{i}} \mathcal{I}_{h} z \mathrm{~d} s+\sum_{j=1}^{N} c_{j} \int_{S_{i}} \varphi_{k_{j}} \mathrm{~d} s=\int_{S_{i}} \mathcal{I}_{h} z \mathrm{~d} s+c_{i} \int_{S_{i}} \varphi_{k_{i}} \mathrm{~d} s=\int_{S_{i}} z \mathrm{~d} s, \quad i=1, \ldots, N,
$$

which amounts to (3.2). 
Now, from assumption (ii) there holds

$$
\left\|\sum_{j=1}^{N} c_{j} \varphi_{k_{j}}\right\|_{\mathrm{H}_{0}^{1}(\mathcal{R})}^{2}=\sum_{j=1}^{N} c_{j}^{2}\left\|\varphi_{k_{j}}\right\|_{\mathrm{H}_{0}^{1}(\mathcal{R})}^{2}
$$

whereas from the definition of $c_{j}$, assumptions (i) and (iv) and (3.4),

$$
\left|c_{j}\right| \leq \frac{\text { length }\left(S_{j}\right)^{1 / 2}}{\underline{L} h_{k_{j}}}\left\|z-\mathcal{I}_{h} z\right\|_{\mathrm{L}^{2}\left(S_{j}\right)} \leq C\|\operatorname{grad} z\|_{\mathrm{L}^{2}\left(\Omega_{k_{j}}\right)} .
$$

Thus, by using that for any regular family of triangulations $\left\|\varphi_{k_{j}}\right\|_{\mathrm{H}_{0}^{1}(\mathcal{R})} \leq C$, we have

$$
\left\|\sum_{j=1}^{N} c_{j} \varphi_{k_{j}}\right\|_{\mathrm{H}_{0}^{1}(\mathcal{R})}^{2} \leq C \sum_{j=1}^{N}\|\operatorname{grad} z\|_{\mathrm{L}^{2}\left(\Omega_{k_{j}}\right)}^{2} \leq C\|z\|_{\mathrm{H}_{0}^{1}(\mathcal{R})}^{2},
$$

the latter because, according to Lemma 3.1, all triangles $T \in \mathcal{T}_{h}$ belong to at most $K$ sets $\Omega_{j}, j=1,2, \ldots, N$. All together yield

$$
\left\|\Pi_{h} z\right\|_{\mathrm{H}_{0}^{1}(\mathcal{R})} \leq\left\|\mathcal{I}_{h} z\right\|_{\mathrm{H}_{0}^{1}(\mathcal{R})}+\left\|\sum_{j=1}^{N} c_{j} \varphi_{k_{j}}\right\|_{\mathrm{H}_{0}^{1}(\mathcal{R})} \leq C\|z\|_{\mathrm{H}_{0}^{1}(\mathcal{R})},
$$

which allows us to end the proof.

Now we are in a position to introduce the discretization of Problem 2.1:

Problem 3.3. Find $\psi_{h} \in \mathcal{Z}_{h}, \lambda_{H} \in \mathcal{M}_{H}$ and $c_{h} \in \mathbb{R}$ such that $\psi_{h}=g$ on $\Gamma$ and

$$
\begin{array}{rlrl}
\int_{\mathcal{R}} \operatorname{grad} \psi_{h} \cdot \operatorname{grad} z_{h} \mathrm{~d} \boldsymbol{x}+\int_{\mathcal{S}} \lambda_{H} z_{h} \mathrm{~d} s & =0 & & \forall z_{h} \in \mathcal{Z}_{h}^{0}, \\
\int_{\mathcal{S}} \mu_{H}\left(\psi_{h}-c_{h}\right) \mathrm{d} s & =0 & \forall \mu_{H} \in \mathcal{M}_{H}, \\
\int_{\mathcal{C}_{R}}\left(\psi_{h}-c_{h}\right) \frac{\partial w_{0}}{\partial \boldsymbol{n}} \mathrm{d} s & =0 . &
\end{array}
$$

To prove that this discrete problem is well posed, we will proceed as in the previous section. With this aim, as a first step, we will analyze the numerical approximation of the auxiliary problems (2.8)-(2.9) and (2.10)-(2.11).

\subsection{Numerical approximation of the auxiliary problems}

Consider the discretization of problems $(2.8)-(2.9)$ and $(2.10)-(2.11)$ obtained by means of the same finite element spaces as above:

- Find $\psi_{1 h} \in \mathcal{Z}_{h}^{0}$ and $\lambda_{1 H} \in \mathcal{M}_{H}$ such that

$$
\begin{aligned}
\int_{\mathcal{R}} \operatorname{grad} \psi_{1 h} \cdot \operatorname{grad} z_{h} \mathrm{~d} \boldsymbol{x}+\int_{\mathcal{S}} \lambda_{1 H} z_{h} \mathrm{~d} s=0 & \forall z_{h} \in \mathcal{Z}_{h}^{0}, \\
\int_{\mathcal{S}} \mu_{H} \psi_{1 h} \mathrm{~d} s=\int_{\mathcal{S}} \mu_{H} \mathrm{~d} s & \forall \mu_{H} \in \mathcal{M}_{H} .
\end{aligned}
$$


- Find $\psi_{2 h} \in \mathcal{Z}_{h}^{0}$ and $\lambda_{2 H} \in \mathcal{M}_{H}$ such that

$$
\begin{aligned}
& \int_{\mathcal{R}} \operatorname{grad} \psi_{2 h} \cdot \operatorname{grad} z_{h} \mathrm{~d} \boldsymbol{x}+\int_{\mathcal{S}} \lambda_{2 H} z_{h} \mathrm{~d} s=-\int_{\mathcal{R}} \operatorname{grad} \psi_{g} \cdot \operatorname{grad} z_{h} \mathrm{~d} \boldsymbol{x} \quad \forall z_{h} \in \mathcal{Z}_{h}^{0}, \\
& \int_{\mathcal{S}} \mu_{H} \psi_{2 h} \mathrm{~d} s=0 \quad \forall \mu_{H} \in \mathcal{M}_{H} .
\end{aligned}
$$

As a consequence of Lemma 3.2 and the standard theory of finite element approximation of mixed problems (see, for instance, [2] or [6]), both problems have a unique solution and the following error estimate holds true:

$$
\left\|\psi_{i}-\psi_{i h}\right\|_{\mathrm{H}_{0}^{1}(\mathcal{R})}+\left\|\lambda_{i}-\lambda_{i H}\right\|_{\mathrm{H}^{1 / 2}(\mathcal{S})^{\prime}} \leq C\left[\inf _{z_{h} \in \mathcal{Z}_{h}}\left\|\psi_{i}-z_{h}\right\|_{\mathrm{H}_{0}^{1}(\mathcal{R})}+\inf _{\mu_{H} \in \mathcal{M}_{H}}\left\|\lambda_{i}-\mu_{H}\right\|_{\mathrm{H}^{1 / 2}(\mathcal{S})^{\prime}}\right], \quad i=1,2 .
$$

In what follows, we will show additional regularity of the solutions of problems (2.8)-(2.9) and (2.10)-(2.11), which together with the estimate above will allow us to obtain an error estimate in terms of adequate powers of the mesh-sizes $h$ and $H$.

The same arguments used in Remark 2.3, allow us to show that $\psi_{1}$ and $\psi_{2}+\psi_{g}$ are harmonic in $\Omega$ and that $\lambda_{i}=-\left[\left[\partial \psi_{i} / \partial \boldsymbol{n}\right]_{\mathcal{S}}, i=1,2\right.$ (recall that $\psi_{2}+\psi_{g}=\psi_{2}$ in a neighborhood of the sail, because $\psi_{g}$ has been chosen vanishing in $\omega \supset \mathcal{S}$ ). Since $\psi_{1}$ and $\psi_{2}+\psi_{g}$ are constant on $\mathcal{S}$ and piecewise linear and continuous on $\Gamma$, both belong to $\mathrm{H}^{1+s}(\Omega)$ for all $s<1 / 2$, because of the standard regularity estimates for the Laplace equation (see [8]). Moreover, each of these two functions takes the same constant values at both sides of the sail $\mathcal{S}$. Therefore,

$$
\psi_{1} \in \mathrm{H}^{1+s}(\mathcal{R}) \quad \text { and } \quad \psi_{2}+\psi_{g} \in \mathrm{H}^{1+s}(\mathcal{R}) \quad \forall s \in\left(0, \frac{1}{2}\right) .
$$

On the other hand, the strongest singularities of $\psi_{1}$ and $\psi_{2}+\psi_{g}$ appear at the end points of the sail, $\boldsymbol{x}_{\mathrm{L}}$ and $\boldsymbol{x}_{\mathrm{T}}$. We focus on the behavior of $\psi_{1}$ around $\boldsymbol{x}_{\mathrm{T}}$, but the same happens for $\psi_{2}+\psi_{g}$, as well as for both functions around $\boldsymbol{x}_{\mathrm{L}}$.

By repeating the arguments leading to (1.13), using the same notation and under the same assumptions, we can write

$$
\psi_{1}=\alpha_{1} \xi+\tilde{\psi}_{1} \quad \text { in } \tilde{\mathcal{B}}_{R}
$$

with $\alpha_{1} \in \mathbb{R}, \xi(r, \theta)=r^{1 / 2} \cos \frac{\theta}{2}$ and $\tilde{\psi} \in \mathrm{W}^{2, p}\left(\tilde{\mathcal{B}}_{R}\right)$ for all $p \in[1,4)$. Explicit computations lead to

$$
\left.\frac{\partial \psi_{1}}{\partial \boldsymbol{n}^{ \pm}}\right|_{\mathcal{S}_{R}^{ \pm}}=-\frac{\alpha_{1}}{2} r^{-1 / 2}+\left.\frac{\partial \tilde{\psi}_{1}}{\partial \boldsymbol{n}^{ \pm}}\right|_{\mathcal{S}_{R}^{ \pm}}
$$

Therefore, the jump of $\partial \psi_{1} / \partial \boldsymbol{n}$ across $\mathcal{S}_{R}$ is given by

$$
\llbracket \frac{\partial \psi_{1}}{\partial \boldsymbol{n}} \rrbracket_{\mathcal{S}_{R}}=\frac{\partial \psi_{1}^{+}}{\partial \boldsymbol{n}^{+}}+\frac{\partial \psi_{1}^{-}}{\partial \boldsymbol{n}^{-}}=-\alpha_{1} r^{-1 / 2}+\llbracket \frac{\partial \tilde{\psi}_{1}}{\partial \boldsymbol{n}} \rrbracket_{\mathcal{S}_{R}} .
$$

Now, the smoothness of $\tilde{\psi}_{1}$ implies that $\left[\partial \tilde{\psi}_{1} / \partial \boldsymbol{n} \rrbracket\right]_{\mathcal{S}_{R}} \in \mathrm{W}^{1-1 / p, p}\left(\mathcal{S}_{R}\right) \hookrightarrow \mathrm{L}^{p}\left(\mathcal{S}_{R}\right)$ for all $p \in[1,4)$. Thus, since $\int_{0}^{R}\left(r^{-1 / 2}\right)^{p} \mathrm{~d} r<\infty$ if and only if $p<2$, we have that $\left.\lambda_{1}\right|_{\mathcal{S}_{R}}=\left[\left[\partial \psi_{1} / \partial \boldsymbol{n}\right]\right]_{\mathcal{S}_{R}} \in \mathrm{L}^{p}\left(\mathcal{S}_{R}\right)$ for all $p \in[1,2)$. The same arguments lead us to analogous conclusions for $\lambda_{1}$ around $\boldsymbol{x}_{\mathrm{L}}$, and for $\lambda_{2}$ around $\boldsymbol{x}_{\mathrm{T}}$ and $\boldsymbol{x}_{\mathrm{L}}$. Thus we conclude that

$$
\lambda_{1}, \lambda_{2} \in \mathrm{L}^{p}(\mathcal{S}) \quad \forall p \in[1,2) .
$$

Next, taking into account the additional regularity (3.13) and (3.14), we will introduce some results about 
approximation by the piecewise polynomial functions involved in the discrete problems. The first one is classical (see, for instance, [4]):

Proposition 3.4. For all $s \in[0,1]$, there exists a constant $C$ such that, for all $z \in \mathrm{H}^{1+s}(\mathcal{R})$,

$$
\inf _{z_{h} \in \mathcal{Z}_{h}}\left\|z-z_{h}\right\|_{\mathrm{H}^{1}(\mathcal{R})} \leq C h^{s}\|z\|_{\mathrm{H}^{1+s}(\mathcal{R})}
$$

To prove an analogous result for the approximation from $\mathcal{M}_{H}$, we introduce the $\mathrm{L}^{2}(\mathcal{S})$-orthogonal projection onto this space. Let $P_{H}: \mathrm{L}^{1}(\mathcal{S}) \rightarrow \mathcal{M}_{H}$ be defined by

$$
P_{H} \varphi:=\sum_{j=1}^{N} \frac{\int_{S_{j}} \varphi \mathrm{d} s}{\operatorname{length}\left(S_{j}\right)} \chi_{j}, \quad \varphi \in \mathrm{L}^{1}(\mathcal{S})
$$

with $\chi_{j}$ being the characteristic function of $S_{j}, j=1, \ldots, N$. (Notice that $\left\{\chi_{j}\right\}_{j=1}^{N}$ is the natural basis of $\mathcal{M}_{H}$.) We have the following estimate:

Lemma 3.5. For all $q \in(2, \infty)$, there exists a constant $C>0$ such that

$$
\left\|\varphi-P_{H} \varphi\right\|_{\mathrm{L}^{q}(\mathcal{S})} \leq C H^{1 / q}\|\varphi\|_{\mathrm{H}^{1 / 2}(\mathcal{S})} \quad \forall \varphi \in \mathrm{H}^{1 / 2}(\mathcal{S})
$$

Proof. For each $S_{j} \in \mathcal{T}_{H}$, let $\boldsymbol{r}_{j}: I:=[0,1] \rightarrow S_{j}$ be a smooth parametrization of the arc $S_{j}$, such that $\left|\boldsymbol{r}_{j}^{\prime}(t)\right|$ is constant for all $t \in I$ (for instance, the affine map which applies $I$ onto the interval $\left[0, \operatorname{length}\left(S_{j}\right)\right]$, $\operatorname{composed}$ with the arc-length parametrization of $\left.S_{j}\right)$. Then, $\left|\boldsymbol{r}_{j}^{\prime}(t)\right|=\int_{0}^{1}\left|\boldsymbol{r}_{j}^{\prime}(t)\right| \mathrm{d} t=\operatorname{length}\left(S_{j}\right)$ for all $t \in I$. Let $\hat{\varphi}:=\varphi \circ \boldsymbol{r}_{j}$ and $\hat{P}$ be the $\mathrm{L}^{2}(I)$-orthogonal projection onto the constant functions. There holds $\hat{P} \hat{\varphi}=\widehat{P_{H} \varphi}:=P_{H} \varphi \circ \boldsymbol{r}_{j}$ and

$$
\left\|\varphi-P_{H} \varphi\right\|_{\mathrm{L}^{q}\left(S_{j}\right)}^{q} \leq \operatorname{length}\left(S_{j}\right)\|\hat{\varphi}-\hat{P} \hat{\varphi}\|_{\mathrm{L}^{q}(I)}^{q} .
$$

Moreover, since both, the canonical injection and the projection $\hat{P}$, are continuous operators from $\mathrm{H}^{1 / 2}(I)$ into $\mathrm{L}^{q}(I)$, we have

$$
\|\hat{\varphi}-\hat{P} \hat{\varphi}\|_{L^{q}(I)}=\|(\hat{\varphi}-d)-\hat{P}(\hat{\varphi}-d)\|_{\mathrm{L}^{q}(I)} \leq C\|\hat{\varphi}-d\|_{\mathrm{H}^{1 / 2}(I)}
$$

for all $d \in \mathbb{R}$, because $\hat{P}(d)=d$. Now, from [4, Theorem 6.1$]$, there exists $d \in \mathbb{R}$ such that

$$
\|\hat{\varphi}-d\|_{\mathrm{H}^{1 / 2}(I)} \leq C|\hat{\varphi}|_{\mathrm{H}^{1 / 2}(I)} \leq C|\varphi|_{\mathrm{H}^{1 / 2}\left(S_{i}\right)},
$$

the last inequality because of the definition of the intrinsic seminorm $|\cdot|_{\mathrm{H}^{1 / 2}(I)}$ (see, for instance, [6], Sect. I.1). Therefore,

$$
\left\|\varphi-P_{H} \varphi\right\|_{\mathrm{L}^{q}(\mathcal{S})} \leq C H^{1 / q}\left[\sum_{i=1}^{N}|\varphi|_{\mathrm{H}^{1 / 2}\left(S_{i}\right)}^{q}\right]^{1 / q} \leq C H^{1 / q}\left[\sum_{i=1}^{N}|\varphi|_{\mathrm{H}^{1 / 2}\left(S_{i}\right)}^{2}\right]^{1 / 2} \leq C H^{1 / q}|\varphi|_{\mathrm{H}^{1 / 2}(\mathcal{S})}
$$

Proposition 3.6. For all $p \in(1,2)$, there exists a constant $C>0$ such that, for all $\mu \in \mathrm{L}^{p}(\mathcal{S})$,

$$
\inf _{\mu_{H} \in \mathcal{M}_{H}}\left\|\mu-\mu_{H}\right\|_{\mathrm{H}^{1 / 2}(\mathcal{S})^{\prime}} \leq C H^{1 / q}\|\mu\|_{\mathrm{L}^{p}(\mathcal{S})}
$$

with $q \in(2, \infty)$ such that $1 / p+1 / q=1$. 
Proof. There holds

$$
\begin{aligned}
\left\|\mu-P_{H} \mu\right\|_{\mathrm{H}^{1 / 2}(\mathcal{S})^{\prime}} & =\sup _{z \in \mathrm{H}_{0}^{1}(\mathcal{R})} \frac{\left\langle\mu-P_{H} \mu, z\right\rangle_{\mathcal{S}}}{\|z\|_{\mathrm{H}_{0}^{1}(\mathcal{R})}}=\sup _{z \in \mathrm{H}_{0}^{1}(\mathcal{R})} \frac{\left\langle\mu-P_{H} \mu, z-P_{H}(z \mid \mathcal{S})\right\rangle_{\mathcal{S}}}{\|z\|_{\mathrm{H}_{0}^{1}(\mathcal{R})}} \\
& \leq \sup _{z \in \mathrm{H}_{0}^{1}(\mathcal{R})} \frac{\left\|\mu-P_{H} \mu\right\|_{\mathrm{L}^{p}(\mathcal{S})}\left\|z-P_{H}\left(\left.z\right|_{\mathcal{S}}\right)\right\|_{\mathrm{L}^{q}(\mathcal{S})}}{\|z\|_{\mathrm{H}_{0}^{1}(\mathcal{R})}} .
\end{aligned}
$$

Thus, the result follows from the previous lemma and the inequality

$$
\left\|\mu-P_{H} \mu\right\|_{L^{p}(\mathcal{S})} \leq\|\mu\|_{L^{p}(\mathcal{S})}+\left\|P_{H} \mu\right\|_{L^{p}(\mathcal{S})} \leq 2\|\mu\|_{L^{p}(\mathcal{S})} .
$$

The above results allow us to obtain the following error estimate for the auxiliary problems:

Proposition 3.7. Let $\left(\psi_{1}, \lambda_{1}\right)$ and $\left(\psi_{2}, \lambda_{2}\right)$ be the solutions of problems (2.8)-(2.9) and (2.10)-(2.11), respectively, and $\left(\psi_{1 h}, \lambda_{1 H}\right)$ and $\left(\psi_{2 h}, \lambda_{2 H}\right)$ the solutions of problems (3.8)-(3.9) and (3.10)-(3.11), respectively. For all $s<1 / 2$ and for all $q>2$, there exists a constant $C>0$ such that

$$
\begin{aligned}
& \left\|\psi_{1}-\psi_{1 h}\right\|_{\mathrm{H}_{0}^{1}(\mathcal{R})}+\left\|\lambda_{1}-\lambda_{1 H}\right\|_{\mathrm{H}^{1 / 2}(\mathcal{S})^{\prime}} \leq C\left[h^{s}\left\|\psi_{1}\right\|_{\mathrm{H}^{1+s}(\mathcal{R})}+H^{1 / q}\left\|\lambda_{1}\right\|_{\mathrm{L}^{p}(\mathcal{S})}\right] \leq C\left(h^{s}+H^{1 / q}\right), \\
& \left\|\psi_{2}-\psi_{2 h}\right\|_{\mathrm{H}_{0}^{1}(\mathcal{R})}+\left\|\lambda_{2}-\lambda_{2 H}\right\|_{\mathrm{H}^{1 / 2}(\mathcal{S})^{\prime}} \leq C\left[h^{s}\left\|\psi_{2}+\psi_{g}\right\|_{\mathrm{H}^{1+s}(\mathcal{R})}+H^{1 / q}\left\|\lambda_{2}\right\|_{\mathrm{L}^{p}(\mathcal{S})}\right] \leq C\left(h^{s}+H^{1 / q}\right),
\end{aligned}
$$

where $1 / p+1 / q=1$.

Proof. The proof follows from (3.12), Propositions 3.4 and 3.6, and the regularity properties (3.13) and (3.14). Notice that for the second inequality above we have also used that, since $\psi_{g}$ has been chosen in $\mathcal{Z}_{h_{0}} \subset \mathcal{Z}_{h}$,

$$
\inf _{z_{h} \in \mathcal{Z}_{h}}\left\|\psi_{2}-z_{h}\right\|_{\mathrm{H}_{0}^{1}(\mathcal{R})}=\inf _{z_{h} \in \mathcal{Z}_{h}}\left\|\psi_{2}+\psi_{g}-z_{h}\right\|_{\mathrm{H}_{0}^{1}(\mathcal{R})} .
$$

\subsection{Numerical approximation of the full problem}

Our next step is to prove that Problem 3.3 is well posed, as long as the meshes are sufficiently fine, and that its solution converges to that of Problem 2.1.

Theorem 3.8. Under the assumption (2.12), there exists $\bar{h}>0$ such that, for all $h<\bar{h}$, Problem 3.3 has a unique solution.

Proof. Existence: Let $\left(\psi_{1 h}, \lambda_{1 H}\right)$ and $\left(\psi_{2 h}, \lambda_{2 H}\right)$ be the solutions of problems (3.8)-(3.9) and (3.10)-(3.11), respectively. From Proposition 3.7, $\left\|\psi_{1}-\psi_{1 h}\right\|_{\mathrm{H}_{0}^{1}(\mathcal{R})} \rightarrow 0$ as $h \rightarrow 0$ and $H \rightarrow 0$. Hence, from (2.12) we deduce the existence of $\bar{h}>0$ and $\gamma>0$ such that

$$
\left|\int_{\mathcal{C}_{R}}\left(\psi_{1 h}-1\right) \frac{\partial w_{0}}{\partial \boldsymbol{n}} \mathrm{d} s\right| \geq \gamma \quad \forall h \leq \bar{h} .
$$

Let us introduce the constant

$$
c_{h}:=-\frac{\int_{\mathcal{C}_{R}} \psi_{2 h} \frac{\partial w_{0}}{\partial \boldsymbol{n}} \mathrm{d} s}{\int_{\mathcal{C}_{R}}\left(\psi_{1 h}-1\right) \frac{\partial w_{0}}{\partial \boldsymbol{n}} \mathrm{d} s} .
$$

Let $\psi_{h}:=c_{h} \psi_{1 h}+\psi_{2 h}+\psi_{g} \in \mathcal{Z}_{h}$ and $\lambda_{H}:=c_{h} \lambda_{1 H}+\lambda_{2 H} \in \mathcal{M}_{H}$. Then, it is straightforward to show that $\left(\psi_{h}, \lambda_{H}, c_{h}\right)$ is a solution of $(3.5)-(3.7)$.

Uniqueness: The proof is essentially identical to that of Theorem 2.5 .

Now we are in a position to prove the convergence of the full discrete problem and an error estimate. 
Theorem 3.9. Let us assume (2.12) and let $\bar{h}>0$ be as in Theorem 3.8. Let $(\psi, \lambda, c)$ be the solution of Problem 2.1. For all $s<1 / 2$ and for all $q>2$, there exists a constant $C>0$ such that, for all $h<\bar{h}$, the solution $\left(\psi_{h}, \lambda_{h}, c_{h}\right)$ of Problem 3.3 satisfies

$$
\left\|\psi-\psi_{h}\right\|_{\mathrm{H}^{1}(\mathcal{R})}+\left\|\lambda-\lambda_{H}\right\|_{\mathrm{H}^{1 / 2}(\mathcal{S})^{\prime}}+\left|c-c_{h}\right| \leq C\left(h^{s}+H^{1 / q}\right) .
$$

Proof. We write $\psi=c \psi_{1}+\psi_{2}+\psi_{g}$ and $\lambda=c \lambda_{1}+\lambda_{2}$, as in the proof of Theorem 2.5, and $\psi_{h}=c_{h} \psi_{1 h}+\psi_{2 h}+\psi_{g}$ and $\lambda_{H}=c_{h} \lambda_{1 H}+\lambda_{2 H}$, as in that of Theorem 3.8. Thus, by virtue of Proposition 3.7, there only remains to prove that $\left|c-c_{h}\right| \leq C\left(h^{s}+H^{1 / q}\right)$.

With this aim, we use (2.13), (3.16) and (3.15), to write for all $h<\bar{h}$

$$
\begin{aligned}
\left|c-c_{h}\right| & =\frac{\left|c \int_{\mathcal{C}_{R}}\left(\psi_{1 h}-\psi_{1}\right) \frac{\partial w_{0}}{\partial \boldsymbol{n}} \mathrm{d} s+\int_{\mathcal{C}_{R}}\left(\psi_{2 h}-\psi_{2}\right) \frac{\partial w_{0}}{\partial \boldsymbol{n}} \mathrm{d} s\right|}{\left|\int_{\mathcal{C}_{R}}\left(\psi_{1 h}-1\right) \frac{\partial w_{0}}{\partial \boldsymbol{n}} \mathrm{d} s\right|} \\
& \leq \frac{C}{\gamma}\left[\left\|\psi_{1}-\psi_{1 h}\right\|_{\mathrm{H}_{0}^{1}(\mathcal{R})}+\left\|\psi_{2}-\psi_{2 h}\right\|_{\mathrm{H}_{0}^{1}(\mathcal{R})}\right] .
\end{aligned}
$$

Thus, the theorem follows from Proposition 3.7, again.

\section{Numerical Results}

The numerical method described above was implemented in a FORTRAN code. This code was applied to solve a couple of test problems with known analytical solutions, which allow us to assess the performance of the method. As a final test, we used it to determine the unknown shape of a sail under the action of the wind by means of an iterative process.

\subsection{Implementation details}

Let $\left\{\varphi_{i}\right\}_{i=1}^{M}$ and $\left\{\chi_{i}\right\}_{i=1}^{N}$ be the respective bases of $\mathcal{Z}_{h}^{0}$ and $\mathcal{M}_{H}$ that have been introduced in Section 3. Let $\psi_{g}$ be as in the previous section $\left(\psi_{g} \in \mathcal{Z}_{h}\right.$ such that $\left.\psi_{g}\right|_{\Gamma}=g$ and it vanishes in a neighborhood of $\left.\mathcal{S} \cup \mathcal{C}_{R}\right)$. We write the solution of Problem 3.3 as follows:

$$
\psi_{h}=\sum_{i=1}^{M} \widehat{\psi}_{i} \varphi_{i}+\psi_{g} \quad \text { and } \quad \lambda_{H}=\sum_{i=1}^{N} \widehat{\lambda}_{i} \chi_{i} .
$$

Let $\widehat{\boldsymbol{\psi}}:=\left(\widehat{\psi}_{i}\right) \in \mathbb{R}^{M}$ and $\widehat{\boldsymbol{\lambda}}:=\left(\widehat{\lambda}_{i}\right) \in \mathbb{R}^{N}$. The matrix form of Problem 3.3 reads:

$$
\left(\begin{array}{ccc}
\boldsymbol{A} & \boldsymbol{B} & \mathbf{0} \\
\boldsymbol{B}^{\mathrm{t}} & \mathbf{0} & \boldsymbol{d} \\
\boldsymbol{s}^{\mathrm{t}} & \mathbf{0} & a
\end{array}\right)\left(\begin{array}{c}
\widehat{\psi} \\
\widehat{\lambda} \\
c_{h}
\end{array}\right)=\left(\begin{array}{l}
\boldsymbol{b} \\
\mathbf{0} \\
0
\end{array}\right)
$$

where $\boldsymbol{A}:=\left(A_{i j}\right) \in \mathbb{R}^{M \times M}, \boldsymbol{B}:=\left(B_{i j}\right) \in \mathbb{R}^{M \times N}, \boldsymbol{b}:=\left(b_{i}\right) \in \mathbb{R}^{M}, \boldsymbol{d}:=\left(d_{i}\right) \in \mathbb{R}^{N}$ and $\boldsymbol{s}:=\left(s_{i}\right) \in \mathbb{R}^{M}$, with

$$
\begin{array}{rlrl}
A_{i j} & :=\int_{\mathcal{R}} \operatorname{grad} \varphi_{i} \cdot \operatorname{grad} \varphi_{j} \mathrm{~d} \boldsymbol{x}, \quad i, j=1, \ldots, M, & d_{i}:=-\int_{\mathcal{S}} \chi_{i} \mathrm{~d} s=\operatorname{length}\left(\mathcal{S}_{i}\right), \quad i=1, \ldots, N, \\
B_{i j} & :=\int_{\mathcal{S}} \varphi_{i} \chi_{j} \mathrm{~d} s=\int_{\mathcal{S}_{j}} \varphi_{i}, \quad i=1, \ldots, M, j=1, \ldots, N, & s_{i} & :=\int_{\mathcal{C}_{R}} \varphi_{i} \frac{\partial w_{0}}{\partial \boldsymbol{n}} \mathrm{d} s, \quad i=1, \ldots, M, \\
b_{i} & :=-\int_{\mathcal{R}} \operatorname{grad} \psi_{g} \cdot \operatorname{grad} \varphi_{i} \mathrm{~d} \boldsymbol{x}, \quad i=1, \ldots, M, & a & :=-\int_{\mathcal{C}_{R}} \frac{\partial w_{0}}{\partial \boldsymbol{n}} \mathrm{d} s=\frac{4}{\sqrt{R}} .
\end{array}
$$




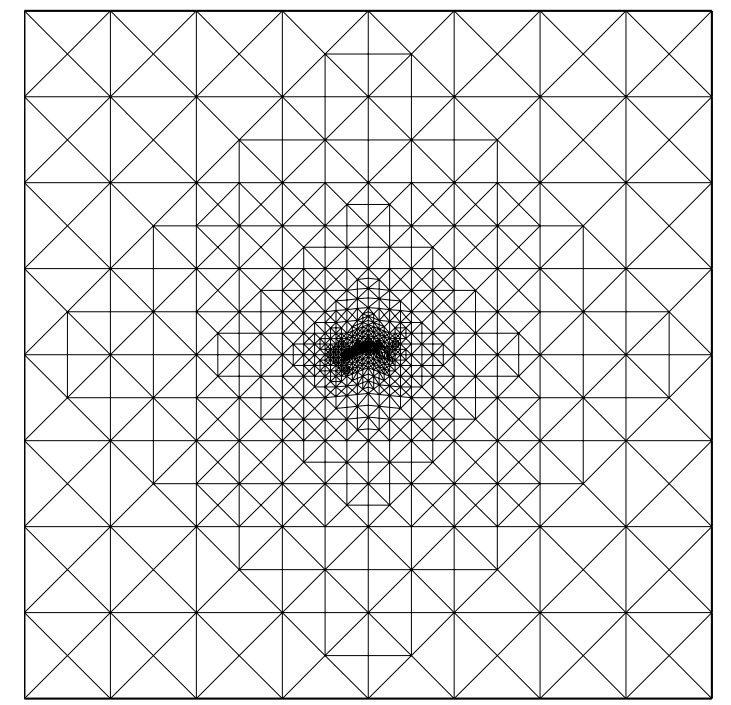

Figure 3. Flat sail. Initial mesh.

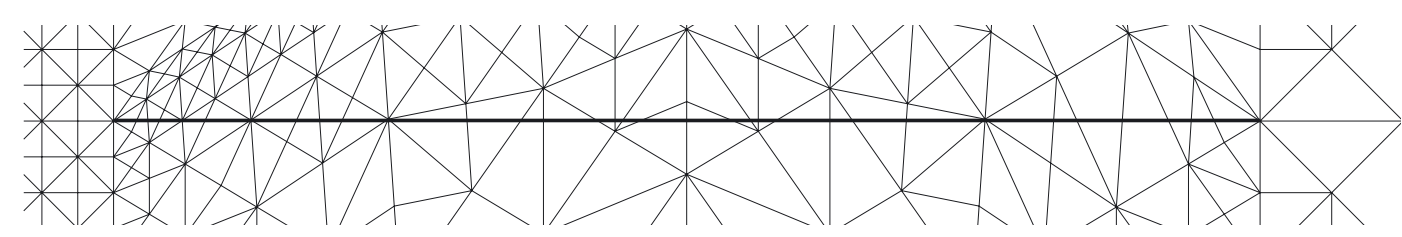

Figure 4. Flat sail. Zoom of the initial mesh around the sail.

The integrals over the circle $\mathcal{C}_{R}$ that define $s_{i}$ have been numerically computed by means of an accurate quadrature rule on each triangle contained in the support of the basis function $\varphi_{i}$. The same happens in the case of a curved sail $\mathcal{S}$ with the integrals over $\mathcal{S}$ defining $B_{i j}$ and $d_{i}$.

\subsection{A flat sail}

The first test consists in computing the irrotational flow past a flat sail. The analytical solution of this problem is well-known (see for instance [1]).

We consider a flat sail $\mathcal{S}=[-5,5] \times\{0\}$ within the domain $\mathcal{R}=[-80,80] \times[-80,80]$. In order to obtain a good approximation at the leading point $\boldsymbol{x}_{\mathrm{L}}=(-5,0)$, we have used the initial mesh shown in Figure 3 which is highly refined in the vicinity of the sail and rather coarser far from it. Subsequent finer meshes were obtained by means of successive uniform refinements of the triangulation shown in this figure.

In this test, it would be easy to use meshes such that the sail be union of edges. However, since we are interested in applying the method to sails with an arbitrary curved shape, we took care to avoid this. Figure 4 shows a zoom of the mesh around the sail where this fact can be clearly observed.

The meshes on the sail were chosen coarse enough so that assumptions (i-iv) from Section 3 were fulfilled. In particular, the initial mesh was taken uniform with only 4 elements. The other ones were obtained by successive uniform refinements of this one.

The main features of the used meshes are reported in Table 1. In particular, it can be seen that the size of the elements in the fluid domain around the sail range between 10 and $60 \%$ the size of the elements on the sail. 
TABLE 1. Flat sail. Data of the used meshes.

\begin{tabular}{|l|c|c|c|c|c|}
\hline Mesh level & 0 & 1 & 2 & 3 & 4 \\
\hline Number of nodes in $\mathcal{S}$ & 5 & 9 & 17 & 33 & 65 \\
Number of elements in $\mathcal{R}$ & 1826 & 7304 & 29216 & 116864 & 467456 \\
Number of nodes in $\mathcal{R}$ & 930 & 3685 & 14673 & 58561 & 233985 \\
Mesh size $h$ on the fluid & 20.0 & 10.0 & 5.0 & 2.5 & 1.25 \\
Smallest diam $(T)$ around the sail & 0.240 & 0.120 & 0.060 & 0.030 & 0.015 \\
Largest diam $(T)$ around the sail & 1.520 & 0.760 & 0.380 & 0.190 & 0.095 \\
Mesh size $H$ on the sail & 2.5 & 1.25 & 0.625 & 0.313 & 0.156 \\
\hline
\end{tabular}

We write the wind velocity at infinity as follows: $v_{\infty}(\cos \alpha, \sin \alpha)$, with $\alpha$ being the angle of incidence. Then, according to [1], the stream function at each point $(x, y) \in \Omega=\mathcal{R} \backslash \mathcal{S}$ is given by

$$
\psi(x, y)=v_{\infty}\left[s \cos \alpha-r \sin \alpha+\frac{a^{2}(r \sin \alpha-s \cos \alpha)}{r^{2}+s^{2}}+2 a \sin \alpha \log \left(\frac{\sqrt{r^{2}+s^{2}}}{a}\right)\right],
$$

where $a:=\operatorname{length}(\mathcal{S}) / 4$,

$$
r:=\frac{x}{2} \pm \frac{\sqrt{2 \sqrt{x^{4}+2 x^{2} y^{2}-8 x^{2} a^{2}+y^{4}+8 y^{2} a^{2}+16 a^{4}}+2\left(x^{2}-y^{2}-4 a^{2}\right)}}{4},
$$

and

$$
s:=\frac{y}{2} \pm \frac{\sqrt{2 \sqrt{x^{4}+2 x^{2} y^{2}-8 x^{2} a^{2}+y^{4}+8 y^{2} a^{2}+16 a^{4}}-2\left(x^{2}-y^{2}-4 a^{2}\right)}}{4},
$$

the signs being chosen so that $r^{2}+s^{2} \geq a^{2}$. After some algebraic manipulations, differentiating $\psi$ leads us to the following expressions for the components of the velocity field $\boldsymbol{v}=\left(v_{1}, v_{2}\right)=\operatorname{curl} \psi$ :

$$
\begin{aligned}
& v_{1}(x, y)=v_{\infty} \frac{\cos \alpha\left(r^{4}+2 r^{2} s^{2}+s^{4}-2 a^{2} r^{2}+2 a^{2} s^{2}+a^{4}\right)+\sin \alpha\left(2 a s r^{2}-4 a^{2} r s+2 a s^{3}+2 a^{3} s\right)}{\left(r^{2}+s^{2}\right)^{2}+a^{4}+2 a^{2}\left(s^{2}-r^{2}\right)}, \\
& v_{2}(x, y)=v_{\infty} \frac{\sin \alpha\left(r^{4}+2 r^{2} s^{2}+s^{4}-2 a r^{3}-2 a r s^{2}-a^{4}+2 a^{3} r\right)}{\left(r^{2}+s^{2}\right)^{2}+a^{4}+2 a^{2}\left(s^{2}-r^{2}\right)} .
\end{aligned}
$$

We chose for this test a wind velocity at infinity corresponding to $\alpha=\pi / 3$ and $v_{\infty}=1$. The radius of the circle $\mathcal{C}_{R}$ to impose the constraint (3.7) was taken $R=0.5$.

The stream function $\psi_{h}$ computed for the level-3 mesh is shown in Figure 5.

The errors of the computed velocity $\boldsymbol{v}_{h}:=\operatorname{curl} \psi_{h}$ in $\mathrm{L}^{2}(\Omega)^{2}$-norm, the Lagrange multiplier $\lambda_{H}$ in $\mathrm{H}^{1 / 2}(\mathcal{S})^{\prime}$ norm and the constant value $c_{h}$ of the stream function on the sail are reported in Table $2^{3}$. Computed orders of convergence in powers of $h$ for all these quantities are also shown in this table. It can be observed that the experimental orders of convergence agree with those predicted by the theory. Actually, a superconvergence phenomenon seems to hold for the constant $c_{h}$, which apparently converges linearly. The table also includes the errors of the circulation around the sail computed as follows (cf. (1.7) and (2.7)):

$$
\gamma_{h}(\mathcal{S}):=-\int_{\mathcal{S}} \lambda_{H} \mathrm{~d} s .
$$

This is a quantity of physical interest and can be seen to converge also with a linear order with respect to $h$.

\footnotetext{
3 The $\mathrm{H}^{1 / 2}(\mathcal{S})^{\prime}$-norm was computed by using the characterization given in Remark 2.2 and approximating problem $(2.4)$ by means of piecewise linear continuous finite elements on a highly refined mesh.
} 

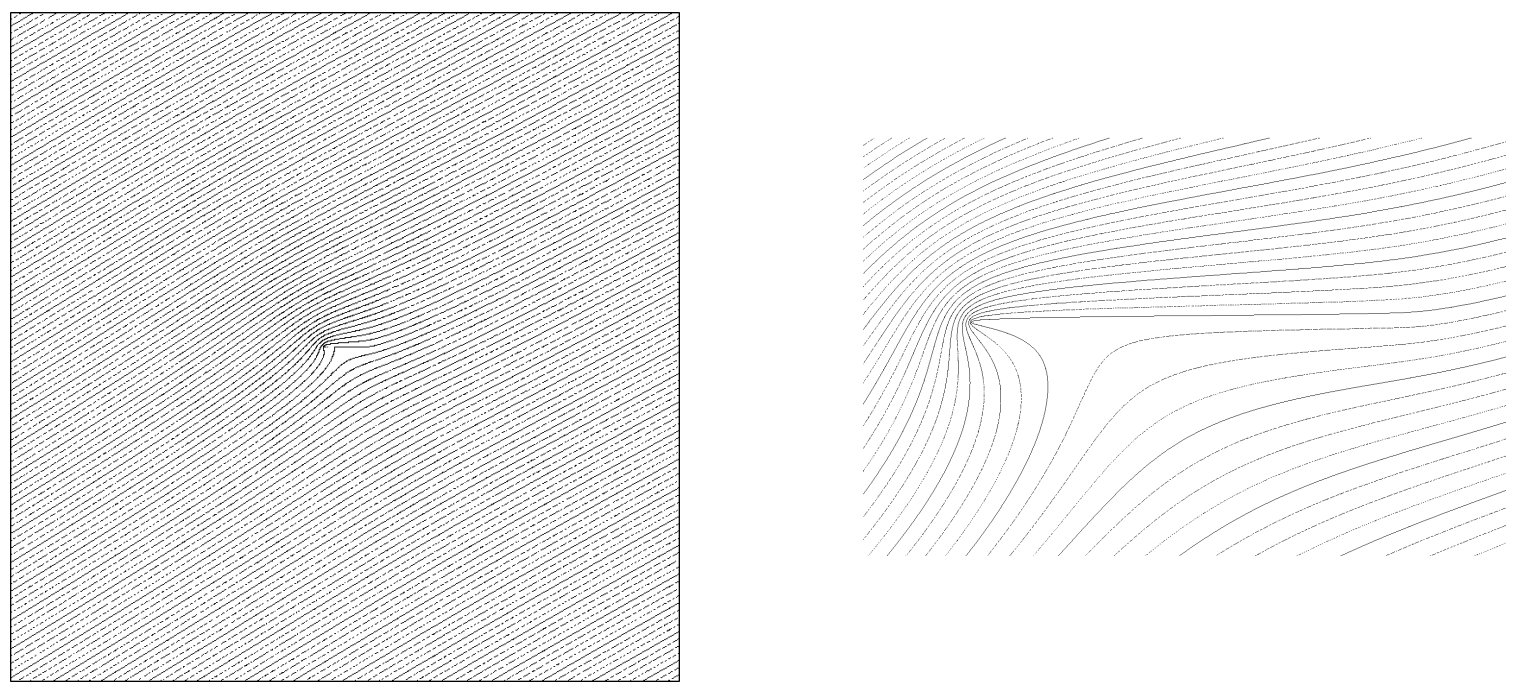

FiguRE 5. Flat sail. Stream function computed on the level-3 mesh (left) and zoom of a more detailed view around the sail (right).

TABLE 2. Flat sail. Errors and convergence order for the velocity field $\boldsymbol{v}_{h}$, the Lagrange multiplier $\lambda_{H}$, the constant value of the stream function on the sail $c_{h}$ and the circulation around the sail $\gamma_{h}(\mathcal{S})$.

\begin{tabular}{|c|c|c|c|c|c|c|c|c|}
\hline $\begin{array}{c}\text { Mesh } \\
\text { level }\end{array}$ & $\frac{\left\|\boldsymbol{v}-\boldsymbol{v}_{h}\right\|_{\left.\mathrm{L}^{2}(\Omega)\right)^{2}}}{\|\boldsymbol{v}\|_{\mathrm{L}^{2}(\Omega)^{2}}}$ & Order & $\frac{\left\|\lambda-\lambda_{H}\right\|_{\mathrm{H}^{1 / 2}(\mathcal{S})^{\prime}}}{\|\lambda\|_{\mathrm{H}^{1 / 2}(\mathcal{S})^{\prime}}}$ & Order & $\left|c-c_{h}\right|$ & Order & $\left|\gamma(\mathcal{S})-\gamma_{h}(\mathcal{S})\right|$ & Order \\
\hline 0 & $0.343 \mathrm{E}-01$ & & $0.177 \mathrm{E}+00$ & & $0.141 \mathrm{E}+01$ & & $0.289 \mathrm{E}+01$ & \\
1 & $0.224 \mathrm{E}-01$ & 0.61 & $0.130 \mathrm{E}+00$ & 0.45 & $0.494 \mathrm{E}+00$ & 1.51 & $0.103 \mathrm{E}+01$ & 1.49 \\
2 & $0.157 \mathrm{E}-01$ & 0.52 & $0.792 \mathrm{E}-01$ & 0.71 & $0.211 \mathrm{E}+00$ & 1.23 & $0.427 \mathrm{E}+00$ & 1.26 \\
3 & $0.111 \mathrm{E}-01$ & 0.50 & $0.577 \mathrm{E}-01$ & 0.46 & $0.978 \mathrm{E}-01$ & 1.11 & $0.200 \mathrm{E}+00$ & 1.10 \\
4 & $0.798 \mathrm{E}-02$ & 0.48 & $0.348 \mathrm{E}-01$ & 0.73 & $0.485 \mathrm{E}-01$ & 1.01 & $0.988 \mathrm{E}-01$ & 1.02 \\
\hline
\end{tabular}

Figures 6-9 show error curves for all these quantities, namely, log-log plots of the errors reported in Table 2 versus the number of elements in $\mathcal{T}_{h}$ (nel).

\subsection{A curved sail}

As a second test we applied the code to compute the irrotational flow past a curved sail. In this case, the sail is an arc of a circle with radius $5 \sqrt{2}$ and central angle $\pi / 2$, the end points of the arc being $\boldsymbol{x}_{\mathrm{L}}=(-5,0)$ and $\boldsymbol{x}_{\mathrm{T}}=$ $(5,0)$. The domain and the wind velocity at infinity were taken as in the previous test: $\mathcal{R}=[-80,80] \times[-80,80]$, $v_{\infty}=1$ and $\alpha=\pi / 3$.

We have used the same meshes as in the previous test for the domain $\mathcal{R}$ and successively refined meshes on the curved sail, the initial one having 6 elements.

Let us remark that this problem does not satisfy thoroughly the assumptions of the theoretical analysis, because the sail is not a straight line in a neighborhood of the trailing point $\boldsymbol{x}_{\mathrm{T}}$. Such a straight line was used in Section 1.1 to define the function $w_{0}$ satisfying (1.15)-(1.16), which in its turn was used to impose the Kutta-Joukowski condition. In spite of this fact, we took $w_{0}$ as defined in $(1.14)$ with $R=0.5$ and $(r, \theta)$ being a polar coordinate system centered at the trailing point $\boldsymbol{x}_{\mathrm{T}}$ and such that the half-line $\theta= \pm \pi$ coincides with the secant to the curved element $S_{i} \in \mathcal{T}_{H}$ containing $\boldsymbol{x}_{\mathrm{T}}$. We have also tried other reasonable choices for this half-line (for instance, the tangent to the $\operatorname{arc} \mathcal{S}$ at $\boldsymbol{x}_{\mathrm{T}}$ ) and the results do not vary significantly. 


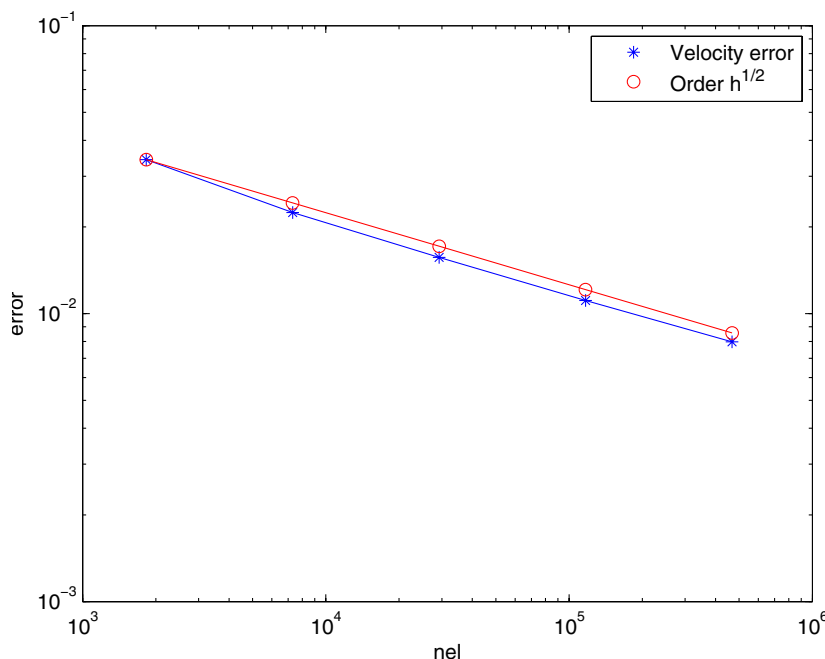

Figure 6. Flat sail. $\quad \mathrm{L}^{2}(\Omega)^{2}$-error curve for the velocity field $\boldsymbol{v}_{h}$.

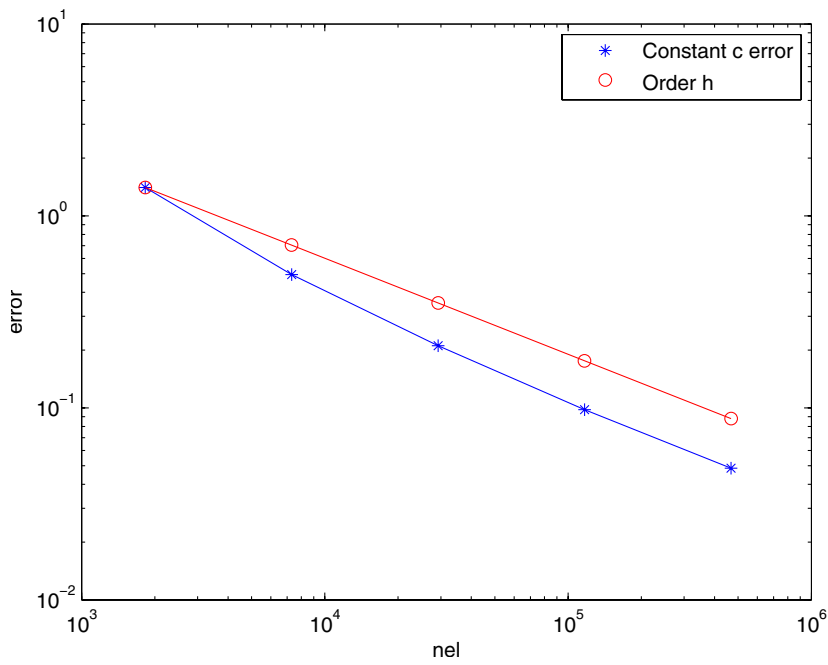

Figure 8. Flat sail. Error curve for the constant value $c_{h}$ of the stream function on the sail.

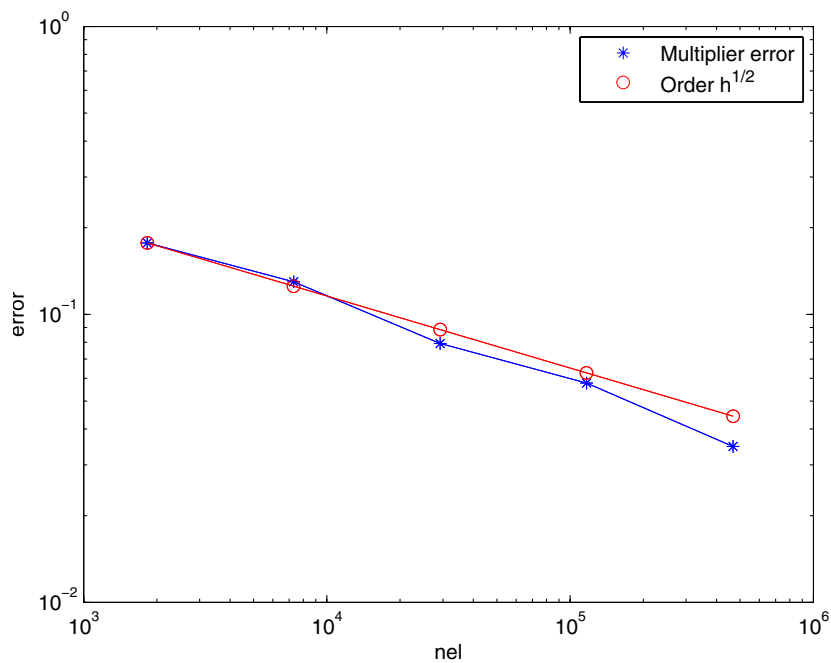

FiguRe 7. Flat sail. $\mathrm{H}^{1 / 2}(\mathcal{S})^{\prime}$-error curve for the Lagrange multiplier $\lambda_{H}$.

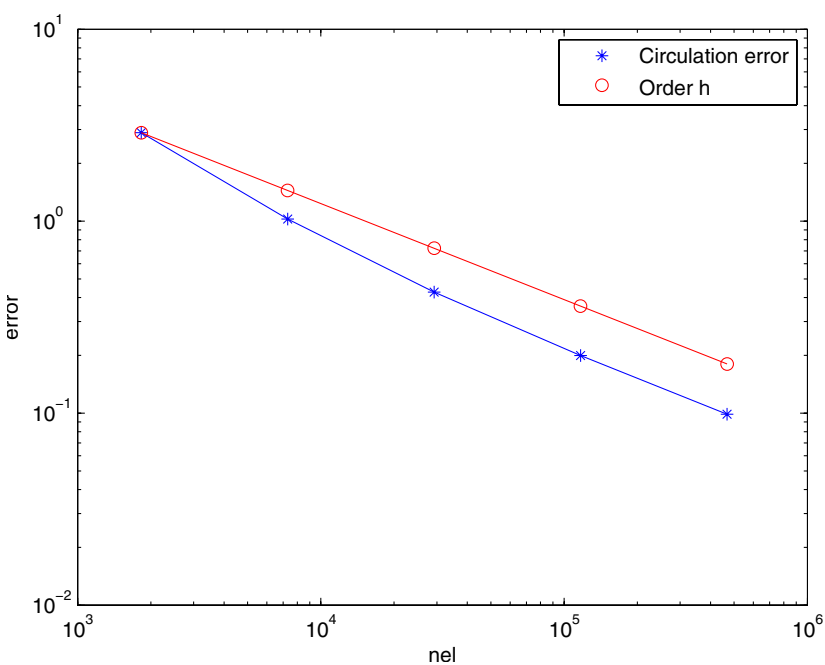

Figure 9. Flat sail. Error curve for the circulation around the sail $\gamma_{h}(\mathcal{S})$.

In this case, the solution is also analytically known (see [1]). We report in Figure 10, Table 3, and Figures 11 to 14 the same results as in the previous test. Once more the numerical results show a good agreement with the theoretical ones and linear orders of convergence are apparent for the constant value of the stream function on the sail and the circulation.

\subsection{Computing the sail shape}

As a final test we applied the numerical method introduced above to solve the fluid-structure interaction problem of determining the shape of a sail under the action of the wind. 

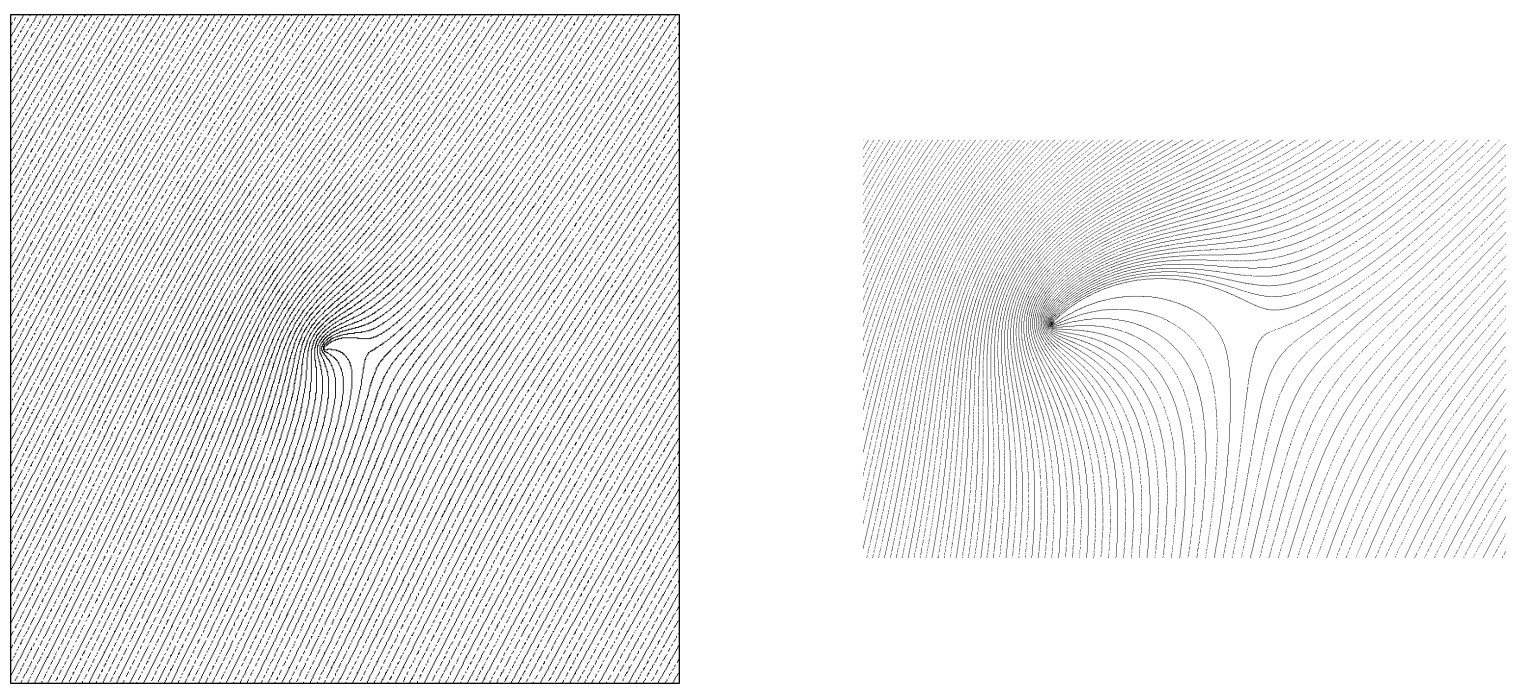

Figure 10. Curved sail. Stream function computed on the level-3 mesh (left) and zoom of a more detailed view around the sail (right).

TABLE 3. Curved sail. Errors and convergence order for the velocity field $\boldsymbol{v}_{h}$, the Lagrange multiplier $\lambda_{H}$, the constant value of the stream function on the sail $c_{h}$ and the circulation around the sail $\gamma_{h}(\mathcal{S})$.

\begin{tabular}{|c|c|c|c|c|c|c|c|c|}
\hline $\begin{array}{c}\text { Mesh } \\
\text { level }\end{array}$ & $\frac{\left\|\boldsymbol{v}-\boldsymbol{v}_{h}\right\|_{\mathrm{L}^{2}(\Omega)^{2}}}{\|\boldsymbol{v}\|_{\mathrm{L}^{2}(\Omega)^{2}}}$ & Order & $\frac{\left\|\lambda-\lambda_{H}\right\|_{\mathrm{H}^{1 / 2}(\mathcal{S})^{\prime}}}{\|\lambda\|_{\mathrm{H}^{1 / 2}(\mathcal{S})^{\prime}}}$ & Order & $\left|c-c_{h}\right|$ & Order & $\left|\gamma(\mathcal{S})-\gamma_{h}(\mathcal{S})\right|$ & Order \\
\hline 0 & $0.223 \mathrm{E}-01$ & & $0.735 \mathrm{E}-01$ & & $0.503 \mathrm{E}+00$ & & $0.116 \mathrm{E}+01$ & \\
1 & $0.143 \mathrm{E}-01$ & 0.64 & $0.548 \mathrm{E}-01$ & 0.42 & $0.270 \mathrm{E}+00$ & 0.90 & $0.584 \mathrm{E}+00$ & 0.99 \\
2 & $0.973 \mathrm{E}-02$ & 0.56 & $0.369 \mathrm{E}-01$ & 0.57 & $0.838 \mathrm{E}-01$ & 1.69 & $0.183 \mathrm{E}+00$ & 1.68 \\
3 & $0.685 \mathrm{E}-02$ & 0.51 & $0.250 \mathrm{E}-01$ & 0.56 & $0.408 \mathrm{E}-01$ & 1.04 & $0.875 \mathrm{E}-01$ & 1.06 \\
4 & $0.486 \mathrm{E}-02$ & 0.50 & $0.152 \mathrm{E}-01$ & 0.71 & $0.170 \mathrm{E}-01$ & 1.26 & $0.371 \mathrm{E}-01$ & 1.24 \\
\hline
\end{tabular}

In accordance to the linear framework that we are using to deal with this problem, we consider a linear string model to compute the position of the sail under the force exerted by the wind flow. More precisely, let us assume that the segment $(0, L)$ is the reference configuration of the sail. A point $x \in(0, L)$ undergoes a displacement in the $y$ direction that we denote $u(x)$, which is the unique solution of the following boundary-value problem:

$$
\begin{aligned}
& -T \frac{\mathrm{d}^{2} u}{\mathrm{~d} x^{2}}(x)=f(x) \quad \forall x \in(0, L), \\
& u(0)=u(L)=0,
\end{aligned}
$$

where $T$ is the tension of the string and $f(x)$ is the force per unit length exerted by the wind on the sail at point $x$.

To compute the force density we make use of Remark 2.3:

$$
\boldsymbol{f} \approx \rho v_{\infty} \lambda \boldsymbol{n}^{-}
$$




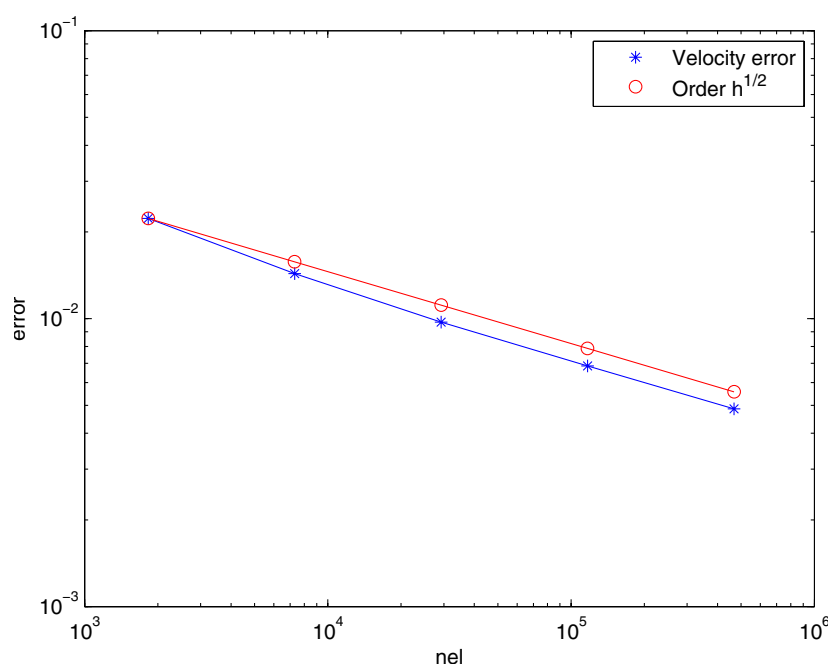

Figure 11. Curved sail. $\mathrm{L}^{2}(\Omega)^{2}$-error curve for the velocity field $\boldsymbol{v}_{h}$.

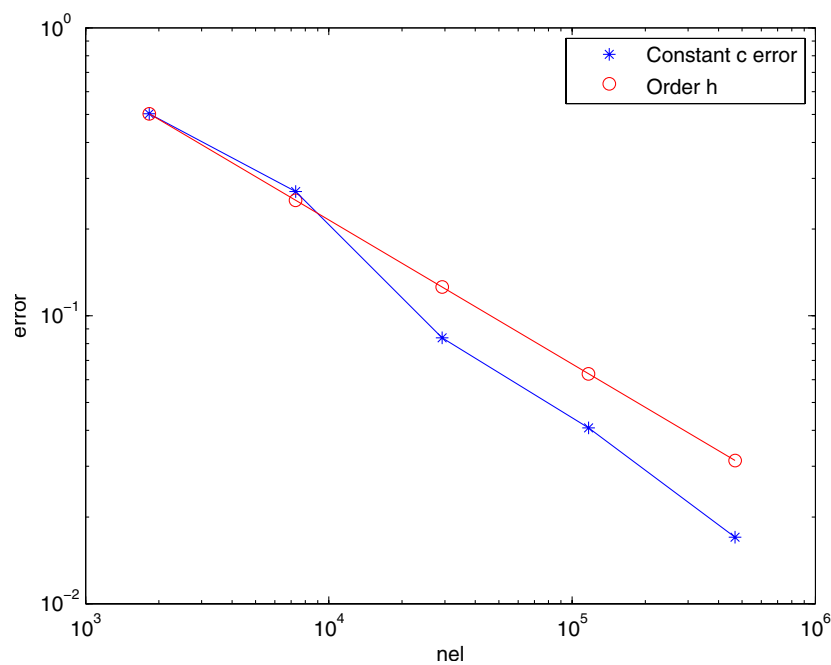

FIGURE 13. Curved sail. Error curve for the constant value $c_{h}$ of the stream function on the sail.

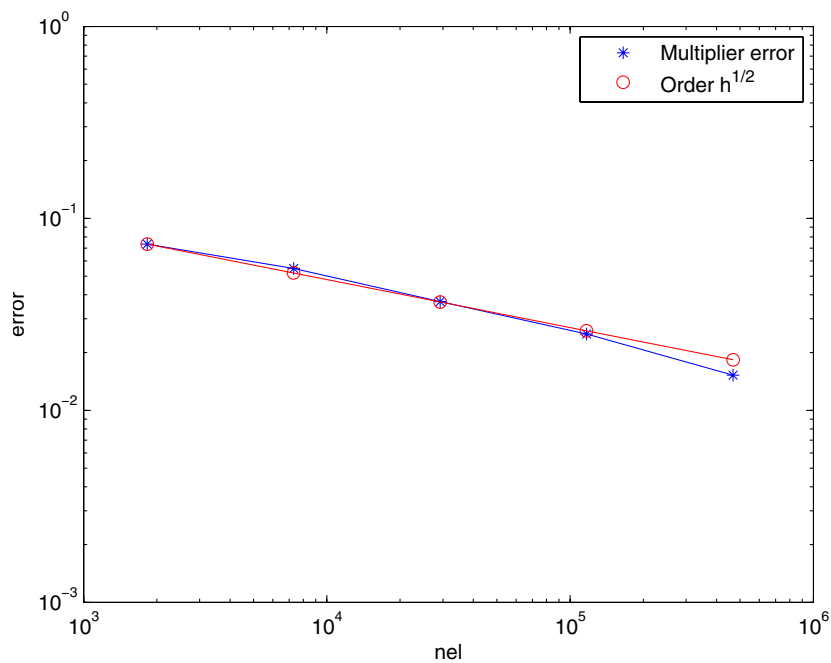

Figure 12. Curved sail. $\mathrm{H}^{1 / 2}(\mathcal{S})^{\prime}$ error curve for the Lagrange multiplier $\lambda_{H}$.

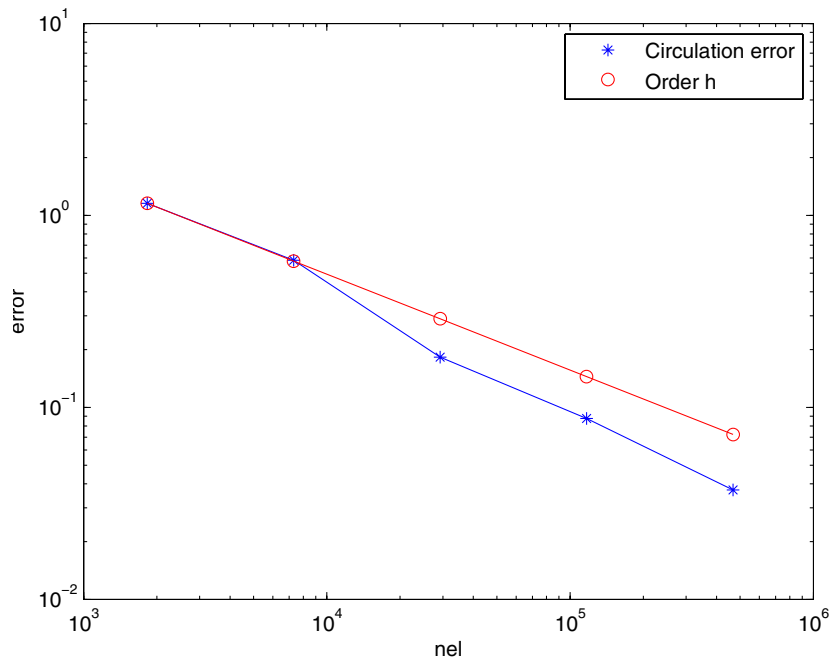

Figure 14. Curved sail. Error curve for the circulation around the sail $\gamma_{h}(\mathcal{S})$.

where $\lambda$ is the Lagrange multiplier arising from Problem 2.1 and $\boldsymbol{n}^{-}$is a unit vector normal to the sail. Recall that the multiplier is evaluated on the deformed sail, which is given by

$$
\mathcal{S}=\{(x, u(x)): x \in(0, L)\}
$$


Moreover, in agreement with the linear theory, we approximate the normal vector $\boldsymbol{n}^{-}$by $\boldsymbol{e}_{2}$. Therefore, we are led to the following fully coupled non-linear problem:

$$
\begin{gathered}
-T \frac{\mathrm{d}^{2} u}{\mathrm{~d} x^{2}}(x)=\rho v_{\infty} \lambda(x, u(x)) \quad \forall x \in(0, L), \\
u(0)=u(L)=0,
\end{gathered}
$$

To solve this problem, we propose a naive fixed point iteration which turns out to be convergent. Starting with the flat sail (i.e., with $\left.u^{(0)}(x) \equiv 0\right)$, the flow problem is solved by using the method described in this paper. Then, we take

$$
f(x)=\rho v_{\infty} \lambda\left(x, u^{(0)}(x)\right)=\rho v_{\infty} \lambda(x, 0), \quad x \in(0, L),
$$

as data in problem (4.1)-(4.2). Since $\lambda$ is piecewise constant, this problem can be easily solved analytically. Thus we obtain its solution, which will be the next iterate $u^{(1)}$. At the following step we use $u^{(1)}$ to compute the new position of the sail and use this to solve again the flow problem. (To impose the Kutta-Joukowski condition, we proceed as in the previous test: we take $w_{0}$ as defined in (1.14) with a polar coordinate system $(r, \theta)$ centered at $\boldsymbol{x}_{\mathrm{T}}$ and such that the half-line $\theta= \pm \pi$ coincides with the secant to the curved element $S_{i} \in \mathcal{T}_{H}$ containing $\boldsymbol{x}_{\mathrm{T}}$.) The process is iterated until convergence. More precisely, the algorithm reads as follows:

\section{Algorithm 4.1.}

- Initialization $(k=0): u^{(0)}:=0$ and $\mathcal{S}^{(0)}:=\{(x, 0): x \in(0, L)\}$.

- Iteration $k \geq 1\left(\mathcal{S}^{(k-1)}\right.$ is known):

- Solve Problem 3.3 for $\mathcal{S}:=\mathcal{S}^{(k-1)}$ to obtain $\psi^{(k)}, \lambda^{(k)}$ and $c^{(k)}$.

- Compute the force exerted by the flow on the sail by

$$
f^{(k)}(x):=\rho v_{\infty} \lambda^{(k)}\left(x, u^{(k-1)}(x)\right) .
$$

- Solve problem (4.1)-(4.2) for $f:=f^{(k)}$ to obtain $u^{(k)}$.

- Define a new position of the sail by

$$
\mathcal{S}^{(k)}:=\left\{\left(x, u^{(k)}(x)\right): x \in(0, L)\right\} .
$$

- Stopping test: $\left\|u^{(k)}-u^{(k-1)}\right\|_{\mathrm{L}^{\infty}(0, L)} \leq \varepsilon$ (a given tolerance)?

The above algorithm was used with the following data:

- domain: $\mathcal{R}=[-80,80] \times[-80,80]$ (lengths in meters);

- wind speed at infinity: $v_{\infty}=5 \mathrm{~m} / \mathrm{s}$;

- angle of incidence: $\alpha=\pi / 6$;

- air density: $\rho=1.2 \mathrm{~kg} / \mathrm{m}^{3}$;

- string tension: $T=1600 \mathrm{~N}$;

- sail length: $L=10 \mathrm{~m}$;

- radius of $\mathcal{C}_{R}: R=0.5 \mathrm{~m}$.

The obtained results are shown in Figures 15 and 16 and Table 4. The final position of the sail (namely, the set $\mathcal{S}$ ) is shown after convergence for different meshes in Figure 15. Furthermore, the different shapes of the sail along the iterations, $\mathcal{S}^{(k)}$, are shown in Figure 16 for a fixed mesh. Finally, the values of the differences between successive iterates for the other physical quantities through the iteration process are reported for the same mesh in Table 4.

Acknowledgements. The authors thank José Manuel Cascón for his help to build the initial mesh for the numerical experiments. 


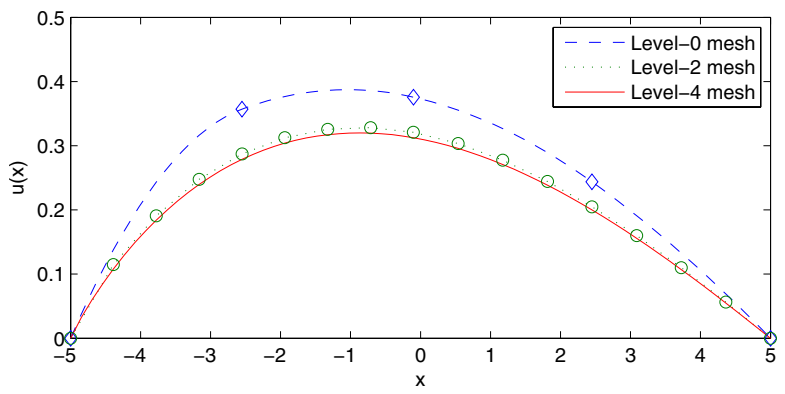

Figure 15. Computing the sail shape. Final sail shape on different meshes.

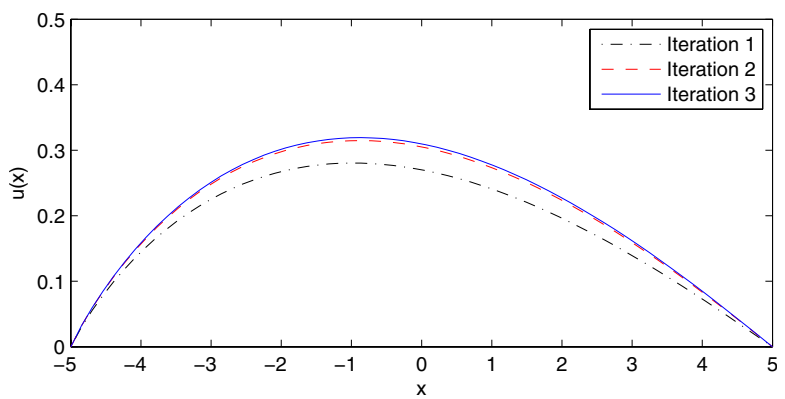

FiguRE 16. Computing the sail shape. Convergence of the sail shape (level-4 mesh).

TABLE 4. Computing the sail shape. Differences between successive iterates for the velocity $\boldsymbol{v}^{(k)}:=\operatorname{curl} \psi^{(k)}$, the Lagrange multiplier $\lambda^{(k)}$ and the sail displacement $u^{(k)}$ (level-4 mesh).

\begin{tabular}{|c|c|c|c|}
\hline $\begin{array}{c}\text { Iteration } \\
\text { number } k\end{array}$ & $\left\|\boldsymbol{v}^{(k)}-\boldsymbol{v}^{(k-1)}\right\|_{\mathrm{L}^{2}(\Omega)^{2}}$ & $\left\|\lambda^{(k)}-\lambda^{(k-1)}\right\|_{\mathrm{H}^{1 / 2}(\mathcal{S})^{\prime}}$ & $\left\|u^{(k)}-u^{(k-1)}\right\|_{\mathrm{L}^{\infty}(0, L)}$ \\
\hline 1 & $0.234 \mathrm{E}+04$ & $0.493 \mathrm{E}+03$ & $0.280 \mathrm{E}+00$ \\
2 & $0.671 \mathrm{E}+02$ & $0.304 \mathrm{E}+01$ & $0.352 \mathrm{E}-01$ \\
3 & $0.962 \mathrm{E}+01$ & $0.713 \mathrm{E}-01$ & $0.462 \mathrm{E}-02$ \\
4 & $0.122 \mathrm{E}+01$ & $0.728 \mathrm{E}-02$ & $0.609 \mathrm{E}-03$ \\
5 & $0.160 \mathrm{E}+00$ & $0.129 \mathrm{E}-03$ & $0.815 \mathrm{E}-04$ \\
6 & $0.214 \mathrm{E}-01$ & $0.235 \mathrm{E}-05$ & $0.109 \mathrm{E}-04$ \\
7 & $0.287 \mathrm{E}-02$ & $0.432 \mathrm{E}-07$ & $0.147 \mathrm{E}-05$ \\
8 & $0.386 \mathrm{E}-03$ & $0.205 \mathrm{E}-08$ & $0.199 \mathrm{E}-06$ \\
\hline
\end{tabular}

\section{REFERENCES}

[1] D.J. Acheson, Elementary Fluid Dynamics. Claredon Press-Oxford (1990).

[2] F. Brezzi and M. Fortin, Mixed and Hybrid Finite Element Methods. Springer-Verlag (1991).

[3] J.F. Ciavaldini, M. Pogu and G. Tournemine, Existence and regularity of stream functions for subsonic flows past profiles with sharp trailing edge. Arch. Rational Mech. Anal. 93 (1986) 1-14.

[4] T Dupont and R. Scott, Polynomial approximation of functions in Sobolev spaces. Math. Comp. 34 (1980) $441-463$.

[5] V. Girault and R. Glowinski, Error analysis of a fictitious domain method applied to a Dirichlet problem. Japan J. Indust. Appl. Math. 12 (1995) 487-514.

[6] V. Girault and P.A. Raviart, Finite Element Methods for Navier-Stokes Equations. Springer-Verlag (1986). 
[7] R. Glowinski, T.W. Pan and J. Périaux, A Lagrange multiplier-fictitious domain method for the Dirichlet problem. Generalization to some flow problems. Japan J. Indust. Appl. Math. 12 (1995) 87-108.

[8] P. Grisvard, Elliptic Problems in Nonsmooth Domains. Pitman (1985).

[9] M.E. Gurtin, An Introduction to Continuum Mechanics. Academic Press (1981).

[10] F. Muttin, A finite element for wrinkled curved elastic membranes, and its application to sails. Comm. Numer. Methods Engrg 12 (1996) 775-785.

[11] N. Parolini and A. Quarteroni, Mathematical models and numerical simulations for the America's Cup. Comput. Methods Appl. Mech. Engrg 194 (2005) 1001-1026.

[12] H. Schoop, Structural and aerodynamic theory for sails. Eur. J. Mech., A/Solids IX (1990) 37-52.

[13] L.R. Scott and S. Zhang, Finite element interpolation of nonsmooth functions satisfying boundary conditions. Math. Comp. 54 (1990) 483-493.

[14] B. Thwaites, The aerodynamic theory of sails. I. Two-dimensional sails. Proc. Roy. Soc. A 261 (1961) 402-422. 